\title{
Search for disappearing tracks as a signature of new long-lived particles in proton-proton collisions at $\sqrt{s}=13 \mathrm{TeV}$
}

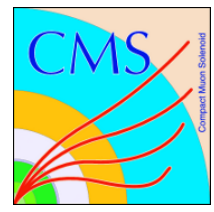

\section{The CMS collaboration}

E-mail: cms-publication-committee-chair@cern.ch

ABSTRACT: A search is presented for long-lived charged particles that decay within the CMS detector and produce the signature of a disappearing track. A disappearing track is an isolated track with missing hits in the outer layers of the silicon tracker, little or no energy in associated calorimeter deposits, and no associated hits in the muon detectors. This search uses data collected with the CMS detector in 2015 and 2016 from protonproton collisions at a center-of-mass energy of $13 \mathrm{TeV}$ at the LHC, corresponding to an integrated luminosity of $38.4 \mathrm{fb}^{-1}$. The results of the search are interpreted in the context of the anomaly-mediated supersymmetry breaking model. The data are consistent with the background-only hypothesis. Limits are set on the product of the cross section for direct production of charginos and their branching fraction to a neutralino and a pion, as a function of the chargino mass and lifetime. At $95 \%$ confidence level, charginos with masses below $715(695) \mathrm{GeV}$ are excluded for a lifetime of $3(7) \mathrm{ns}$, as are charginos with lifetimes from 0.5 to $60 \mathrm{~ns}$ for a mass of $505 \mathrm{GeV}$. These are the most stringent limits using a disappearing track signature on this signal model for chargino lifetimes above $\approx 0.7 \mathrm{~ns}$.

Keywords: Beyond Standard Model, Hadron-Hadron scattering (experiments)

ArXiv EPrint: 1804.07321 


\section{Contents}

1 Introduction 1

2 The CMS detector 2

3 Data sets 3

4 Event reconstruction and selection 4

5 Background estimation $\quad 7$

5.1 Charged leptons 7

$\begin{array}{ll}5.2 \text { Spurious tracks } & 10\end{array}$

6 Systematic uncertainties $\quad 10$

$\begin{array}{ll}6.1 \text { Background estimates } & 10\end{array}$

$\begin{array}{lll}6.2 & \text { Signal efficiencies } & 12\end{array}$

$\begin{array}{lll}7 & \text { Results } & 12\end{array}$

$\begin{array}{lll}8 & \text { Summary } & 13\end{array}$

$\begin{array}{ll}\text { The CMS collaboration } & 21\end{array}$

\section{Introduction}

This paper presents a search for long-lived, charged particles that decay within the volume of the silicon tracker of the CMS detector at the CERN LHC and produce the signature of a "disappearing track." A disappearing track occurs when the decay products of a charged particle are undetected because they either have too little momentum to be reconstructed or interact only weakly, such that they do not produce hits in the tracker and do not deposit significant energy in the calorimeters.

Anomaly-mediated supersymmetry breaking (AMSB) [1,2] is one of the many beyondthe-standard-model (BSM) scenarios in which such a disappearing track would be produced, and one that has been widely used to interpret the results of searches for disappearing tracks. In AMSB, a particle mass spectrum is predicted with a small mass difference between the lightest chargino $\left(\widetilde{\chi}_{1}^{ \pm}\right)$and neutralino $\left(\widetilde{\chi}_{1}^{0}\right)$, where the latter is the lightest supersymmetric particle [3-6]. The chargino decays to a neutralino and a pion: $\widetilde{\chi}_{1}^{ \pm} \rightarrow \widetilde{\chi}_{1}^{0} \pi^{ \pm}$. Because of the small chargino-neutralino mass difference, the phase space for this decay is limited, and as a consequence the chargino has a lifetime on the order of 1 ns. The pion from this decay has low momentum $(\approx 100 \mathrm{MeV})$, generally too low for it to be observable 
as a reconstructed track. If the chargino decays inside the tracker volume, it thus will often leave a disappearing track. We present the search in terms of the chargino mass and lifetime in AMSB, although other BSM scenarios that produce a disappearing track signature have also been proposed [3, 7-11].

Previous analyses performed by the CMS and ATLAS collaborations have searched for disappearing tracks in proton-proton (pp) collision data at $\sqrt{s}=8 \mathrm{TeV}[12,13]$, and a recent analysis by the ATLAS collaboration searched for short disappearing tracks in $13 \mathrm{TeV}$ data [14]. The previous CMS search excluded at 95\% confidence level (CL) direct electroweak production of charginos with a mass less than $505 \mathrm{GeV}$ for a mean proper lifetime of $7 \mathrm{~ns}$, while the ATLAS search at $13 \mathrm{TeV}$ extended the exclusion limits on chargino mass to $460 \mathrm{GeV}$ for a lifetime of $0.2 \mathrm{~ns}$. These searches are complementary to searches for heavy stable charged particles, which are able to exclude charginos with much longer lifetimes $[15,16]$. Two significant improvements with respect to the $8 \mathrm{TeV}$ search for disappearing tracks have been implemented for this search at $13 \mathrm{TeV}$ : a new dedicated trigger developed specifically for this search, and an estimation of the background from standard model (SM) leptons entirely based on control samples in data.

\section{The CMS detector}

The central feature of the CMS apparatus is a superconducting solenoid of $6 \mathrm{~m}$ internal diameter. Within the solenoid volume are a silicon pixel and strip tracker, a lead tungstate crystal electromagnetic calorimeter (ECAL), and a brass and scintillator hadron calorimeter (HCAL), each composed of a barrel and two endcap sections. Forward calorimeters extend the pseudorapidity coverage provided by the barrel and endcap detectors. Muons are measured in gas-ionization detectors embedded in the steel flux-return yoke outside the solenoid.

The silicon tracker measures charged particles within the pseudorapidity range $|\eta|<$ 2.5. It consists of 1440 silicon pixel and 15148 silicon strip detector modules and is located in the $3.8 \mathrm{~T}$ field of the solenoid. For particles that are not explicitly required to be isolated from other event activity, and that have transverse momentum $1<p_{\mathrm{T}}<10 \mathrm{GeV}$ and $|\eta|<1.4$, the track resolutions are typically $1.5 \%$ in $p_{\mathrm{T}}$ and $25-90(45-150) \mu \mathrm{m}$ in the transverse (longitudinal) impact parameter. These particles represent the bulk of those produced in collisions. For comparison, isolated particles of $p_{\mathrm{T}}=100 \mathrm{GeV}$ emitted at $|\eta|<1.4$ have track resolutions of $2.8 \%$ in $p_{\mathrm{T}}$ and $10(30) \mu \mathrm{m}$ in the transverse (longitudinal) impact parameter [17].

Events of interest are selected using a two-tier trigger system [18]. The first level (L1), composed of custom hardware processors, uses information from the calorimeters and muon detectors to select events at a rate of around $100 \mathrm{kHz}$ within a time interval of less than $4 \mu \mathrm{s}$. The second level, known as the high-level trigger (HLT), consists of a farm of processors running a version of the full event reconstruction software optimized for fast processing, and reduces the event rate to around $1 \mathrm{kHz}$ before data storage.

A more detailed description of the CMS detector, together with a definition of the coordinate system used and the relevant kinematic variables, can be found in ref. [19]. 


\begin{tabular}{|lc|}
\hline Run period & Integrated luminosity $\left[\mathrm{fb}^{-1}\right]$ \\
\hline 2015 & 2.7 \\
$2016 \mathrm{~A}$ & 8.3 \\
$2016 \mathrm{~B}$ & 27.4 \\
\hline
\end{tabular}

Table 1. The data-taking periods and the corresponding integrated luminosities.

\section{Data sets}

This search uses pp collision data corresponding to an integrated luminosity of $38.4 \mathrm{fb}^{-1}[20$, 21], collected with the CMS detector at $\sqrt{s}=13 \mathrm{TeV}$ during 2015 and 2016 . We analyze separately the data collected during each of the two years. Further, because of changes to the trigger configuration during the 2016 run, we also consider the earlier and later data-taking periods, designated as 2016A and 2016B, separately. The three running periods, which we analyze independently, and the corresponding integrated luminosities are presented in table 1.

Simulated signal events of $\mathrm{pp} \rightarrow \widetilde{\chi}_{1}^{ \pm} \widetilde{\chi}_{1}^{\mp}$ and $\mathrm{pp} \rightarrow \widetilde{\chi}_{1}^{0} \widetilde{\chi}_{1}^{ \pm}$are generated at leading order (LO) precision with PYTHIA 6.4.26 [22] with the CTEQ6L1 [23] parton distribution function (PDF) set for $\widetilde{\chi}_{1}^{ \pm}$masses from 100 to $900 \mathrm{GeV}$ and lifetimes from $0.33 \mathrm{~ns}$ to $330 \mathrm{~ns}$, using sparticle mass spectra produced by ISAJET 7.80 [24]. The branching fraction for $\widetilde{\chi}_{1}^{ \pm} \rightarrow \widetilde{\chi}_{1}^{0} \pi^{ \pm}$ is set to $100 \%$, and $\tan \beta$ is fixed to 5 with $\mu>0$, where $\tan \beta$ is the ratio of the vacuum expectation values of the two Higgs doublets and $\mu$ is the higgsino mass parameter. In practice the $\widetilde{\chi}_{1}^{ \pm}-\widetilde{\chi}_{1}^{0}$ mass difference has little dependence on $\tan \beta$ and the sign of $\mu$ [25]. These events are normalized using chargino production cross sections calculated at next-toleading order (NLO) plus next-to-leading-logarithmic (NLL) precision using RESUMmiNO 1.0.9 [26, 27] with CTEQ6.6 [28] and MSTW2008nlo90cl [29] PDF sets. The final cross sections and uncertainties are calculated using the PDF4LHC recommendations for the two sets of cross sections [30]. The ratio of $\widetilde{\chi}_{1}^{0} \widetilde{\chi}_{1}^{ \pm}$to $\widetilde{\chi}_{1}^{ \pm} \widetilde{\chi}_{1}^{\mp}$ production is estimated to be roughly 2:1 for all chargino masses considered. Scale factors are applied as a function of the $p_{\mathrm{T}}$ of the sparticle pair (either $\widetilde{\chi}_{1}^{ \pm} \widetilde{\chi}_{1}^{\mp}$ or $\widetilde{\chi}_{1}^{0} \widetilde{\chi}_{1}^{ \pm}$) to correct for mismodeling of initial state radiation (ISR) in PYTHIA; they are derived by comparing experimental and simulated data in a control region populated mainly by $\mathrm{Z} \rightarrow \mu \mu$ decays as a function of the $p_{\mathrm{T}}$ of the $\mathrm{Z}$ boson candidate, similar to the method used in ref. [31]. These events were chosen because the production modes of the $\mathrm{Z}$ boson and the $\widetilde{\chi}_{1}^{ \pm}$are similar. The scale factors typically result in a correction of order $+25 \%$ in the kinematic region relevant to this search.

Although the methods used to estimate backgrounds in this search are based on experimental data, samples of simulated SM processes are used to validate them and calculate systematic uncertainties. Drell-Yan events, single top quark production via the $s$ and $t$ channels, $\mathrm{Z} \gamma, \mathrm{W} \gamma$, and $\mathrm{W} \rightarrow \ell \nu$ events, where $\ell$ can be an electron, muon, or tau lepton, are generated at NLO precision using the MADGRAPH5_aMC@NLO 2.3.3 generator [32]. The WZ, ZZ, and quantum chromodynamics (QCD) multijet events, with the last composed of jets produced solely through the strong interaction, are generated at LO precision

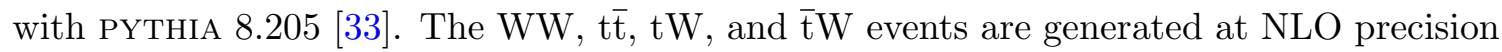


using POWHEG v2.0 [34-40]. The fragmentation and hadronization for all simulated background processes are provided by PYTHIA 8.205. The NNPDF3.0 [41] PDF set is used for all simulated backgrounds, and the CUETP8M1 $[42,43]$ tune is used for the underlying event.

For both simulated signal and background events, the detector response is described by a full model of the CMS detector based on GEANT4 [44] and reconstructed with the same software that is used for collision data. Simulated minimum bias events are superimposed on the hard interaction to describe the effect of overlapping inelastic pp interactions within the same or neighboring bunch crossings, known as pileup, and the samples are reweighted to match the reconstructed vertex multiplicity observed in data.

\section{Event reconstruction and selection}

The particle-flow (PF) event algorithm [45] is designed to reconstruct and identify each individual particle with an optimized combination of information from the various elements of the CMS detector. The energy of photons is directly obtained from the ECAL measurement, corrected for zero-suppression effects. The energy of electrons is determined from a combination of the electron momentum at the primary interaction vertex as determined by the tracker, the energy of the corresponding ECAL cluster, and the energy sum of all bremsstrahlung photons spatially compatible with originating from the electron track. The energy of muons is obtained from the curvature of the corresponding track. The energy of charged hadrons is determined from a combination of their momentum measured in the tracker and the matching ECAL and HCAL energy deposits, corrected for zerosuppression effects and for the response function of the calorimeters to hadronic showers. Finally, the energy of neutral hadrons is obtained from the corresponding corrected ECAL and HCAL energy.

This search is performed on events that pass one or more of several triggers with requirements on missing transverse momentum, a characteristic of signal events where the missing transverse momentum is generated by an ISR jet recoiling off the sparticle pair. For this specific analysis we define the vector $\vec{p}_{\mathrm{T}}^{\text {miss }}$, with magnitude $p_{\mathrm{T}}^{\text {miss }}$, as the projection onto the plane perpendicular to the beam axis of the negative vector sum of the momenta of all reconstructed PF candidates in an event, with the exception of muons, or, in the case of the $\mathrm{L} 1$ trigger, of all calorimeter energy deposits. The triggers require $p_{\mathrm{T}}^{\text {miss }}$ at L1, with the specific requirement varying throughout data taking with changes in the instantaneous luminosity. At the HLT, events with either $p_{\mathrm{T}}^{\text {miss }}$ or $p_{\mathrm{T}}^{\text {miss, } \mu}$, which is defined similarly to $p_{\mathrm{T}}^{\text {miss }}$ but with muons included in its calculation, are selected. The lowestthreshold trigger, which was developed specifically for this search, requires $p_{\mathrm{T}}^{\text {miss }}>75 \mathrm{GeV}$ as well as an isolated track with $p_{\mathrm{T}}>50 \mathrm{GeV}$ at the HLT. The higher-threshold triggers require either $p_{\mathrm{T}}^{\text {miss }}$ or $p_{\mathrm{T}}^{\text {miss, } \mu}$ to be greater than 90 (120) GeV for the 2015 (2016) data. For signal events, which typically have no reconstructed muons, $p_{\mathrm{T}}^{\text {miss }}$ and $p_{\mathrm{T}}^{\mathrm{miss}, \mu}$ are usually identical, and both are used at the HLT to mitigate any inefficiency in the isolated track

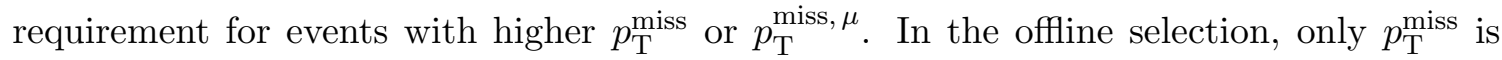
used, in order to mirror the requirements in the L1 trigger and lowest-threshold HLT path. 
Events are required to have $p_{\mathrm{T}}^{\text {miss }}>100 \mathrm{GeV}$ offline, where $p_{\mathrm{T}}^{\text {miss }}$ is calculated from the full $\mathrm{PF}$ reconstruction.

Jets are clustered from PF candidates using FASTJET 3.10 [46] with the anti- $k_{\mathrm{T}}$ algorithm [47] with a distance parameter of 0.4 , and only jets with $p_{\mathrm{T}}>30 \mathrm{GeV}$ and $|\eta|<2.4$ are considered in the analysis. Additional criteria are imposed on these jets to remove those originating from calorimeter noise and misidentified leptons [48]. Events are required to have at least one jet with $p_{\mathrm{T}}>110 \mathrm{GeV}$ in order to be consistent with the ISR recoil topology.

We require the difference in azimuthal angle, $\phi$, between the $\vec{p}_{\mathrm{T}}$ of the leading (highest energy) jet and $\vec{p}_{\mathrm{T}}^{\text {miss }}$ to be greater than 0.5 , and for events with at least two jets, we require the maximum difference in $\phi$ between any two jets, $\Delta \phi_{\max }$, to be less than 2.5. These requirements are designed to remove the large, reducible background originating from QCD multijet events. In these events, a dijet topology with back-to-back jets dominates and mismeasurement of the jet energy may result in a significant measured $p_{\mathrm{T}}^{\text {miss }}$. We refer to the selection up to this point, before any track-related criteria are imposed, as the "basic selection." Events passing this selection are expected to have minimal signal contamination and are dominated by the $\mathrm{W} \rightarrow \ell \nu$ process. The effect of the two angular requirements of the basic selection on the 2016 data and on simulated signal and background events is shown in figure 1. For signal events, the shapes of these distributions are largely independent of chargino mass and lifetime, and a single representative signal point is shown. The combination of these two requirements is sufficient to remove most of the large QCD multijet background that would otherwise pass the basic selection, while the majority of the remaining background is removed by the track criteria described below.

After the basic selection, tracks are selected that have $p_{\mathrm{T}}>55 \mathrm{GeV}$ and $|\eta|<2.1$. The track $p_{\mathrm{T}}$ requirement is chosen such that the corresponding requirement in the HLT path is fully efficient. We ensure that selected tracks are isolated from other activity in the tracker by requiring the scalar sum of the $p_{\mathrm{T}}$ of other tracks within a cone of $\Delta R=\sqrt{(\Delta \phi)^{2}+(\Delta \eta)^{2}}<0.3$ around the momentum vector of the selected track be less than $5 \%$ of the $p_{\mathrm{T}}$ of the track. Selected tracks are also required to be well-separated from jets with $\Delta R$ (track, jet) $>0.5$.

One source of background for this search arises from "spurious tracks," i.e., pattern recognition errors that do not correspond to actual charged particles. Spurious tracks can have missing hits in the outer layers of the silicon tracker and muon detectors, and are not generally associated with large energy deposits in the calorimeters, thus mimicking a disappearing track. This background is suppressed by requiring that selected tracks have at least three hits in the pixel detector and at least seven hits overall in the tracker, a typical non-disappearing track leaving twice that number of hits on average. A missing hit in a layer of the tracker between the interaction point and the first actual hit on the track is called a missing inner hit, while a missing hit between the first and last hits on the track is called a missing middle hit. We require selected tracks to have no missing inner or middle hits. In other words, there must be a consecutive pattern of hits originating in the tracker layers closest to the interaction point. Since spurious tracks often appear displaced from the interaction point, we also require all tracks to have a transverse impact 

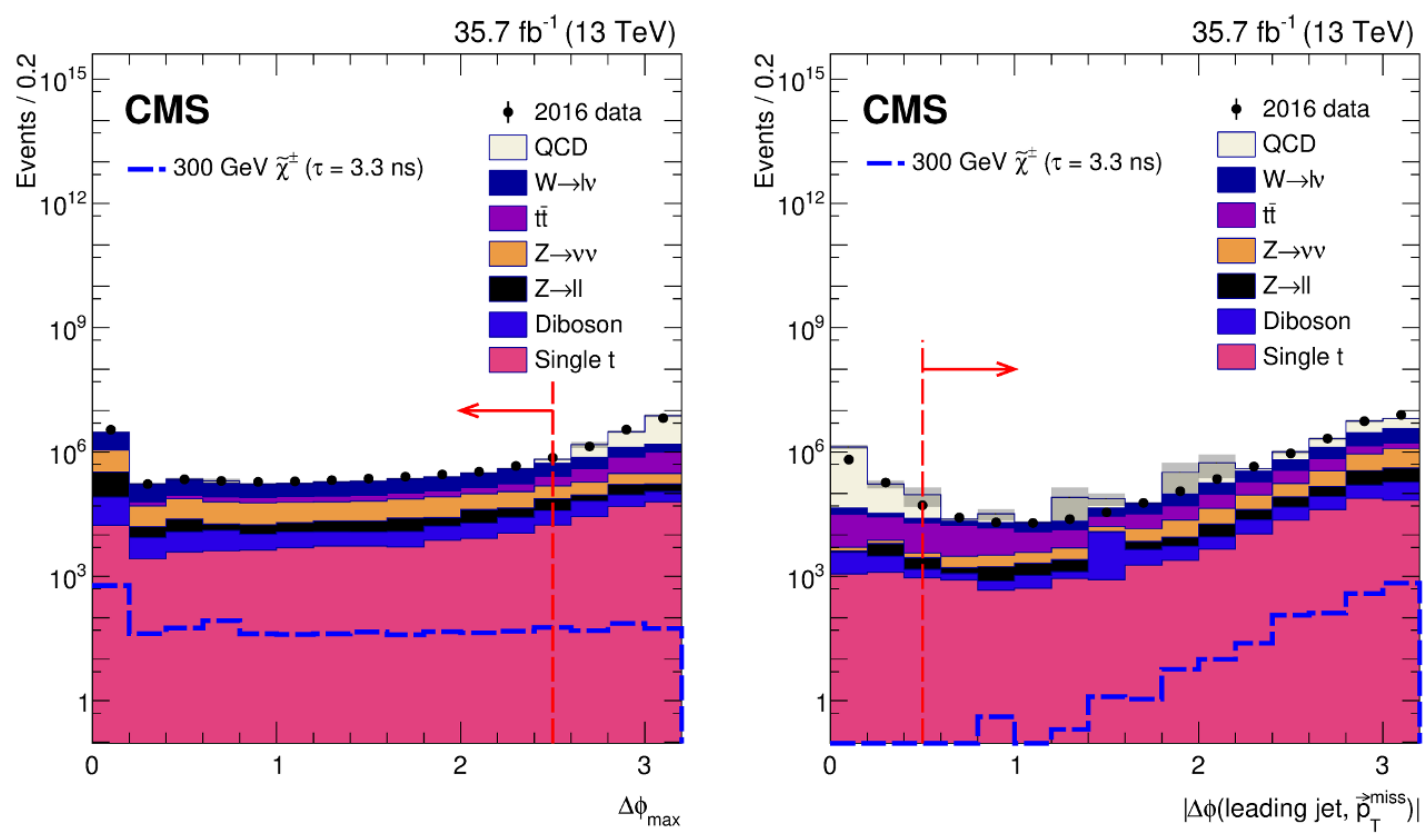

Figure 1. Distributions of the maximum difference in $\phi$ between any two jets (left) and the difference in $\phi$ between the $\vec{p}_{\mathrm{T}}$ of the leading jet and $\vec{p}_{\mathrm{T}}^{\text {miss }}$ (right) for events passing the basic selection, before either of the requirements on these two variables is imposed. The data is from the 2016 data-taking period, and the blue dashed lines show the distributions for simulated signal events with a chargino that has a lifetime of $3.3 \mathrm{~ns}$ and mass of $300 \mathrm{GeV}$, with a corresponding production cross section of $0.58 \mathrm{pb}$. The gray shaded area indicates the statistical uncertainty in the SM background, and the leftmost bin of the left plot includes events with only one selected jet. The vertical dashed lines indicate the chosen value for the requirement on each variable, and the arrows indicate which events are selected.

parameter $\left|d_{0}\right|<0.02 \mathrm{~cm}$ and a longitudinal impact parameter $\left|z_{0}\right|<0.5 \mathrm{~cm}$, both with respect to the primary vertex, chosen as the reconstructed vertex with the largest value of summed physics object $p_{T}^{2}$. The physics objects are the jets, clustered using the jet finding algorithm [46, 47] with the tracks assigned to the vertex as inputs, and the associated missing transverse momentum, taken as the negative vector sum of the $p_{\mathrm{T}}$ of those jets. More details are given in section 9.4.1 of ref. [49].

Besides spurious tracks, most isolated, high- $p_{\mathrm{T}}$ tracks from SM processes come from charged leptons produced in the decays of $\mathrm{W}$ or $\mathrm{Z}$ bosons or virtual photons. Thus, the other main source of background for this search arises from isolated charged leptons that are not correctly reconstructed by the PF algorithm. Leptons can have missing hits in the tracker for several reasons: for example, energetic bremsstrahlung in the case of electrons, or nuclear interactions with the tracker material in the case of hadronically decaying tau leptons $\left(\tau_{\mathrm{h}}\right)$. Leptons may also have small associated calorimeter energy deposits because of nonoperational or noisy channels. To mitigate this background, events where selected tracks are close to reconstructed leptons $(\Delta R$ (track, lepton $)<0.15)$ are vetoed. To avoid selecting leptons that fail to be reconstructed because of detector inefficiencies, we impose the following fiducial track criteria. We avoid regions of muon reconstruction inefficiency 
by vetoing tracks within gaps in the coverage of the muon chambers at $0.15<|\eta|<0.35$ and $1.55<|\eta|<1.85$. Similarly, we avoid regions of electron reconstruction inefficiency by rejecting tracks within the overlap region between the barrel and endcap sections of the ECAL at $1.42<|\eta|<1.65$, as well as tracks within $\Delta R<0.05$ of a nonoperational or noisy ECAL channel, where $\Delta R$ is calculated with respect to the track at the point of closest approach to the center of CMS.

Additional areas of inefficiency are identified using electron and muon tag-and-probe $(\mathrm{T} \& \mathrm{P})$ studies [50], where $\mathrm{Z} \rightarrow \ell \ell$ candidates are selected in data with $m_{\ell \ell} \approx m_{\mathrm{Z}}$, where $m_{\mathrm{Z}}$ is the world-average mass of the $\mathrm{Z}$ boson [51], and the $\mathrm{Z}$ resonance is exploited to obtain a sample of tracks that have a high probability of being leptons, without explicitly requiring them to be reconstructed as leptons. The fraction of these tracks that are not explicitly identified as leptons passing a loose set of identification criteria is a measure of the inefficiency for identifying leptons and is grouped in bins in the $\eta-\phi$ plane. Tracks in bins with an anomalously high inefficiency are rejected from the selection. This procedure removes $\approx 4 \%$ of tracks in simulated signal events that would otherwise be selected.

Two additional requirements define the criteria for a track to "disappear." First, we require the selected tracks to have at least three missing outer hits, which are missing hits in the tracker layers outside of the last layer containing a hit on the track. Second, the associated calorimeter energy within $\Delta R<0.5$ of the track, $E_{\text {calo }}$, is required to be less than $10 \mathrm{GeV}$, where $\Delta R$ is calculated using the track coordinates at the point of closest approach to the center of CMS. This requirement removes a negligible amount of signal, while $E_{\text {calo }}$ is much larger, typically over $100 \mathrm{GeV}$, for background events passing the other selection criteria, according to the simulation. The number of missing outer hits is shown in figure 2 for simulated signal and background events that pass the full selection, except for the requirement on that variable. The tracks selected in the simulated background events are predominantly from electrons and $\tau_{\mathrm{h}}$, since events with muons have a smaller $p_{\mathrm{T}}^{\text {miss }}$ on average. As can be seen, the number of missing outer hits is very effective at isolating the signal because tracks from background events typically have no missing outer hits. The efficiency of the full selection for simulated signal events is limited mostly by the requirements targeting events with ISR and the relatively narrow range of chargino decay lengths that yield a disappearing track that passes the criterion on the number of missing outer hits. This efficiency varies with the chargino mass and lifetime, peaking at $\approx 2 \%$ for a $700 \mathrm{GeV}$ chargino with a lifetime of $3 \mathrm{~ns}$.

\section{Background estimation}

\subsection{Charged leptons}

The dominant source of high- $p_{\mathrm{T}}$, isolated tracks from SM processes arises from charged leptons (electrons and muons, produced promptly or via the decay of tau leptons, or $\tau_{\mathrm{h}}$ ) from the decay of $\mathrm{W}$ or $\mathrm{Z}$ bosons or virtual photons. In order for events with such tracks to appear in the search region, three things must happen: (1) the lepton must fail to be explicitly identified as a lepton, while still leaving a track in the silicon tracker but less than $10 \mathrm{GeV}$ of energy in the calorimeters; (2) the resulting $p_{\mathrm{T}}^{\text {miss, } \mu}$ and $p_{\mathrm{T}}^{\text {miss }}$ must be large 


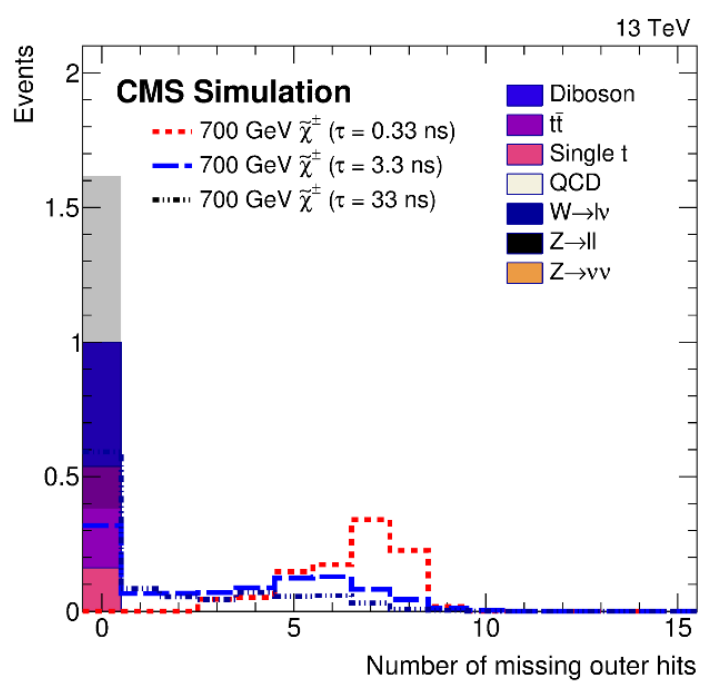

Figure 2. Distributions of the number of missing outer hits for tracks in simulation that pass the full selection, except for the requirement on that variable. Each signal distribution and the sum of the SM background distributions are scaled to have unit area. The gray shaded area indicates the statistical uncertainty in the SM background.

enough for the event to pass the triggers; and (3) the resulting $p_{\mathrm{T}}^{\text {miss }}$ must be large enough for the event to pass the offline $p_{\mathrm{T}}^{\text {miss }}$ requirements.

The key point is that $p_{\mathrm{T}}^{\text {miss, } \mu}$ and $p_{\mathrm{T}}^{\text {miss }}$ are affected by whether the lepton is explicitly identified as a lepton or not. If it is not, but still leaves a track in the silicon tracker and less than $10 \mathrm{GeV}$ of energy in the calorimeters, its energy does not typically contribute to the visible energy of the event. The method used to estimate the background from charged leptons is based on calculating the probability in data of the three conditions listed above, with each lepton flavor treated independently.

The first probability we consider is $P_{\text {veto }}$, the probability that the lepton in a singlelepton event is not explicitly identified as a lepton. For each flavor of charged lepton, we estimate $P_{\text {veto }}$ using a T\&P study. The electron (muon) T\&P selections utilize $\mathrm{Z} \rightarrow$ ee $(\mu \mu)$ candidates. For this study we select events passing a single-electron (single-muon) trigger and containing a reconstructed electron (muon) that passes tight identification and isolation criteria. This lepton serves as the tag. A probe track is required to pass the disappearing track criteria, except for those defining the electron (muon) veto in table 2 . The tag lepton and the probe track are required to have an invariant mass within $10 \mathrm{GeV}$ of the $\mathrm{Z}$ boson mass and to have opposite signs of electric charge.

For the $\tau_{\mathrm{h}} \mathrm{T} \& \mathrm{P}$ study, we define two selections using $\mathrm{Z} \rightarrow \tau \tau$ events that are combined for the calculation of $P_{\text {veto }}$ : one where the electron from a $\tau \rightarrow$ e $\nu \nu$ candidate is selected as the tag, and one where the muon from a $\tau \rightarrow \mu \nu \nu$ candidate is selected as the tag. These two selections are identical to the electron and muon T\&P selections defined above, respectively, except for two modifications. First, we require the transverse mass $m_{\mathrm{T}}=\sqrt{2 p_{\mathrm{T}}^{\ell} p_{\mathrm{T}}^{\text {miss, } \mu}(1-\cos \Delta \phi)}$ to be less than $40 \mathrm{GeV}$, where $p_{\mathrm{T}}^{\ell}$ is the magnitude of the transverse momentum of the tag lepton and $\Delta \phi$ is the difference in $\phi$ between the $\vec{p}_{\mathrm{T}}$ of 


\begin{tabular}{|lccc|}
\hline Selection & Electron & Muon & Tau lepton \\
\hline Min $\Delta R_{\text {track, electron }}>0.15$ & $\checkmark$ & & \\
Min $\Delta R_{\text {track, muon }}>0.15$ & & $\checkmark$ & \\
Min $\Delta R_{\text {track, } \tau_{\mathrm{h}}>0.15}$ & & & $\checkmark$ \\
$E_{\text {calo }}<10 \mathrm{GeV}$ & $\checkmark$ & & $\checkmark$ \\
Missing outer hits $\geq 3$ & $\checkmark$ & $\checkmark$ & $\checkmark$ \\
$\Delta R_{\text {track, jet }}>0.5$ & & & $\checkmark$ \\
\hline
\end{tabular}

Table 2. Definitions of the lepton vetoes used in the T\&P studies to estimate $P_{\text {veto }}$, for each flavor of charged lepton. The criteria listed are the subset of the search criteria that are the most efficient at rejecting each flavor.

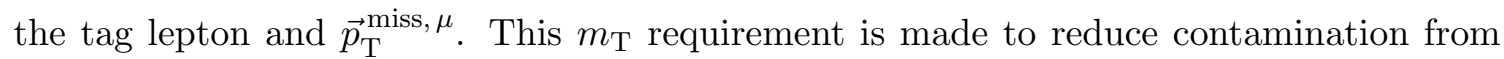
$\mathrm{W} \rightarrow \ell \nu$ events. Second, because the $\tau$ leptons from the $\mathrm{Z}$ decay are not fully reconstructed, the dilepton invariant mass requirement is $m_{\mathrm{Z}}-50<m<m_{\mathrm{Z}}-15 \mathrm{GeV}$.

For each of these selections, we also define a version in which the tag lepton and the probe track are required to have the same sign of electric charge instead of opposite signs. This requirement makes it unlikely that the selected probe track candidates are genuine tracks, and these selections are used to subtract the background from spurious tracks in the calculation of $P_{\text {veto }}$.

For each of the three T\&P channels (electrons, muons, and $\tau_{\mathrm{h}}$ ), the quantities $N_{\mathrm{T} \& \mathrm{P}}$ $\left(N_{\text {T\&P }}^{\text {veto }}\right)$ and $N_{\text {SS T\&P }}\left(N_{\text {SS T\&P }}^{\text {veto }}\right)$ are the numbers of selected T\&P pairs before (after) the final lepton veto is applied to the probe tracks, for the opposite-sign and same-sign selection, respectively. From this, the veto probability is calculated as:

$$
P_{\text {veto }}=\frac{N_{\mathrm{T} \& \mathrm{P}}^{\text {veto }}-N_{\mathrm{SST} \text { T\&P }}^{\text {veto }}}{N_{\mathrm{T} \& \mathrm{P}}-N_{\mathrm{SST} \text { T\& }}}
$$

We define $P_{\text {offline }}$ as the conditional probability of a single-lepton event to pass the offline requirements of $p_{\mathrm{T}}^{\text {miss }}>100 \mathrm{GeV}$ and $\mid \Delta \phi$ (leading jet, $\left.\vec{p}_{\mathrm{T}}^{\text {miss }}\right) \mid>0.5$ given that the lepton candidate is not explicitly identified as a lepton. Using events in single-lepton control regions in data, we introduce a modified $\vec{p}_{\mathrm{T}}^{\text {miss }}$ variable that represents what $\vec{p}_{\mathrm{T}}^{\text {miss }}$ would look like if the lepton in these events were not explicitly identified as such, assuming that if a lepton is not explicitly identified it contributes no visible energy to the event. In the single-electron and single $\tau_{\mathrm{h}}$ control regions, we use $\vec{p}_{\mathrm{T}}^{\text {miss }}+\vec{p}_{\mathrm{T}}^{\text {lepton }}$. For the single-muon control region, we simply use $\vec{p}_{\mathrm{T}}^{\text {miss }}$ since the $p_{\mathrm{T}}$ of all reconstructed muons is already excluded from its calculation. We then estimate $P_{\text {offline }}$ by counting the fraction of events with $p_{\mathrm{T}}^{\text {miss }}>100 \mathrm{GeV}$ and $\mid \Delta \phi$ (leading jet, $\vec{p}_{\mathrm{T}}^{\text {miss }}$ ) $\mid>0.5$ after modifying $\vec{p}_{\mathrm{T}}^{\text {miss }}$ in this way.

We define $P_{\text {trigger }}$ as the conditional probability of a single-lepton event to pass the $p_{\mathrm{T}}^{\text {miss, } \mu}$ or $p_{\mathrm{T}}^{\text {miss }}$ triggers, given that the lepton candidate is not explicitly identified as a lepton and that the event passes the offline requirements of $p_{\mathrm{T}}^{\text {miss }}>100 \mathrm{GeV}$ and $\mid \Delta \phi$ (leading jet, $\left.\vec{p}_{\mathrm{T}}^{\text {miss }}\right) \mid>0.5$. The estimation of $P_{\text {trigger }}$ is made in a similar way to the estimation of $P_{\text {offline }}$ in the single-lepton control regions, assuming that a lepton that is not explicitly identified as such contributes no visible energy to the event and constructing the modified $\vec{p}_{\mathrm{T}}^{\text {miss }}+\vec{p}_{\mathrm{T}}^{\text {lepton }}$ for electrons and $\tau_{\mathrm{h}}$, using $\vec{p}_{\mathrm{T}}^{\text {miss }}$ for muons. The exception for 
$P_{\text {trigger }}$ is that instead of constructing these quantities with offline reconstructed leptons, online objects are used from both the L1 trigger and the HLT. For each lepton selected in each of the single-lepton control regions in data, we find the closest L1 trigger object and closest HLT object within $\Delta R<0.1$ of the offline object. The $\vec{p}_{\mathrm{T}}$ of these objects is then added to the nominal $\vec{p}_{\mathrm{T}}^{\text {miss }}$, as calculated by the L1 trigger and HLT, respectively, and to the nominal $\vec{p}_{\mathrm{T}}^{\text {miss, } \mu}$ in the case of the HLT. This way, we can test, event by event, if the L1 trigger and HLT would have passed, given these modifications to the online $\vec{p}_{\mathrm{T}}^{\text {miss, } \mu}$ and $\vec{p}_{\mathrm{T}}^{\text {miss }}$. The number of events passing the offline $p_{\mathrm{T}}^{\text {miss }}$ requirements is calculated following the procedure used to calculate $P_{\text {offline }}$, and the fraction of these events that also pass the $p_{\mathrm{T}}^{\text {miss, } \mu}$ and $p_{\mathrm{T}}^{\text {miss }}$ triggers according to the above procedure is then the estimate of $P_{\text {trigger }}$.

The product of the three probabilities defined above $\left(P_{\text {veto }}, P_{\text {offline }}\right.$, and $\left.P_{\text {trigger }}\right)$ gives the probability of an event with a charged lepton to enter the search region. We use the single-lepton control regions to estimate the total numbers of events in data containing each flavor of lepton, $N_{\text {ctrl }}^{\ell}$, and obtain the estimated number of background events from charged leptons as

$$
N_{\mathrm{est}}^{\ell}=N_{\mathrm{ctrl}}^{\ell} P_{\text {veto }} P_{\text {offline }} P_{\text {trigger }} .
$$

Closure tests were performed with samples of simulated background events and with the early $13 \mathrm{TeV}$ data taken in 2015. Both tests proved the validity of the background estimation method, with agreement within $1.2 \sigma$ observed in all cases.

\subsection{Spurious tracks}

The contribution of spurious tracks to the background is largely suppressed by the requirement that the impact parameters of the tracks with respect to the primary vertex are small and by the requirement that the tracks are missing no inner or middle hits in the tracker. We estimate the residual contribution from this background using a control region of $\mathrm{Z} \rightarrow \mu \mu$ events as a representative sample of SM events. Within this sample, we additionally require a track, separate from the muons coming from the $\mathrm{Z}$ boson candidate, that passes the track requirements of the search region except for the transverse impact parameter criterion, which we replace with a sideband selection, $0.02<\left|d_{0}\right|<0.10 \mathrm{~cm}$, designed to enhance the likelihood that the tracks we select are spurious. In this way, we can estimate the probability for there to be spurious tracks that satisfy these requirements. This probability is multiplied by a transfer factor to obtain the probability of spurious tracks passing the nominal impact parameter requirement, $P_{\text {spurious }}$. This transfer factor is obtained from a sample of tracks with only three hits in the pixel detector and no hits in the strip detector, which is dominated by spurious tracks. The estimated background from spurious tracks is the number of events in data passing the basic selection, $N_{\mathrm{ctrl}}^{\text {basic }}$, multiplied by $P_{\text {spurious }}$ :

$$
N_{\mathrm{est}}^{\text {spurious }}=N_{\mathrm{ctrl}}^{\text {basic }} P_{\text {spurious }}
$$

\section{$6 \quad$ Systematic uncertainties}

\subsection{Background estimates}

The lepton background estimates rely on the assumption that when a lepton is not explicitly identified as a lepton, while still leaving a track in the silicon tracker but less than $10 \mathrm{GeV}$ 
of energy in the calorimeters, it contributes no visible energy to the event. We test the impact of this assumption for electrons and $\tau_{\mathrm{h}}$ by replacing the nominal $\vec{p}_{\mathrm{T}}^{\text {miss }}+\vec{p}_{\mathrm{T}}^{\text {lepton }}$ variable used to calculate $P_{\text {offline }}$ and $P_{\text {trigger }}$ with a "scaled down" version,

$$
\vec{p}_{\mathrm{T}}^{\text {miss }}+\frac{p_{\mathrm{T}}^{\text {lepton }}-10 \mathrm{GeV}}{p_{\mathrm{T}}^{\text {lepton }}} \vec{p}_{\mathrm{T}}^{\text {lepton }},
$$

and recalculating $P_{\text {offline }}$ and $P_{\text {trigger }}$. In other words, we assume that unreconstructed leptons contribute $10 \mathrm{GeV}$ of visible energy to the event. The value of $10 \mathrm{GeV}$ is chosen because selected tracks are required to have $E_{\text {calo }}<10 \mathrm{GeV}$ in the disappearing track search region. The difference from unity of the ratio

$$
\frac{\left(P_{\text {offline }} P_{\text {trigger }}\right)_{\text {scaled down }}}{\left(P_{\text {offline }} P_{\text {trigger }}\right)_{\text {nominal }}}
$$

is taken as the systematic uncertainty. This uncertainty is approximately $12(17) \%$ for electrons $\left(\tau_{\mathrm{h}}\right)$ and is not calculated for muons, since even successfully reconstructed muons are not expected to contribute substantial visible calorimeter energy to an event.

For the spurious track background estimate, it is assumed that the particular choice of the $d_{0}$ sideband region results in predominantly spurious tracks being selected. To test the impact of this assumption, we examine the variations in the background estimate as the lower bound on the sideband is increased from 0.02 to $0.10 \mathrm{~cm}$. These variations are indeed consistent with the nominal estimate within statistical uncertainties, with maximum variations of $100 \%$ down and $45 \%$ up for the 2016 data, which are assigned as systematic uncertainties. For the 2015 data, since the estimate is zero and there is no indication of behavior different from 2016 data, we assign a systematic uncertainty of $50 \%$ for this data. To apply systematic uncertainties to estimates of zero events, the recommendations of ref. [52] are followed.

A systematic uncertainty associated with the evaluation of the sideband transfer factor using tracks with three hits is determined. This systematic uncertainty is evaluated by examining the variation in the $d_{0}$ distribution from tracks with three consecutive hits to at least seven consecutive hits using tracks in simulated events that are not associated with a generated particle. In this way, we can see how much the true distribution of $d_{0}$ for spurious tracks varies with the number of hits, and constrain the impact this variation has on the background estimate. This procedure yields an uncertainty of approximately $-50 \%$ and $+100 \%$ in the spurious-track background estimate.

The spurious-track background estimate rests on the assumption that the spurioustrack probability is similar for events in the $\mathrm{Z} \rightarrow \mu \mu$ control region and events passing the basic selection. However, there is nothing about the method used to calculate this probability that prevents us from calculating it for events passing the basic selection, and we are able to compare the estimates we obtain from these two independent control regions. This comparison serves to validate the method for estimating the spurious-track background, and the relative difference between the estimates is assigned as a systematic uncertainty. Excellent agreement is seen between the two control regions in both the spurious-track probability and the spurious-track estimate itself, with the estimates agreeing to within 


\begin{tabular}{|lc|}
\hline Source of uncertainty & Range [\%] \\
\hline Theory & $3-9$ \\
Integrated luminosity & $2.3-2.5$ \\
Pileup & $2-3$ \\
ISR & $8-9$ \\
Jet energy scale/resolution & $2-6$ \\
$p_{\mathrm{T}}^{\text {miss modeling }}$ & 0.4 \\
Missing inner hits & $1-3$ \\
Missing middle hits & $0.3-3$ \\
Missing outer hits & $0-3$ \\
$E_{\text {calo }}$ selection & $0.6-1$ \\
Trigger efficiency & $4-6$ \\
Track reconstruction efficiency & $1.5-4.5$ \\
Total & $10-18$ \\
\hline
\end{tabular}

Table 3. Summary of the systematic uncertainties in the signal yields. The ranges represent either the variation with chargino mass and lifetime or with the data-taking period used to calculate the uncertainty, depending on the source of each uncertainty as described in the text.

$\approx 8 \%$ for the 2016 data, and this is taken as a systematic uncertainty. Again, both estimates are zero in the 2015 data, but without any indication that their behaviors are different from 2016 data, we assign a $20 \%$ systematic uncertainty for this period and implement this as in ref. [52].

\subsection{Signal efficiencies}

Theoretical uncertainties of 3-9\% (depending on the chargino mass), which include factorization and renormalization scale uncertainties as well as the PDF uncertainties, are assigned to the chargino production cross sections. Additional sources of systematic uncertainty in the signal yields include those in the integrated luminosity, $2.3(2.5) \%$ for 2015 (2016) data [20, 21], and those related to the modeling of pileup (2-3\%), ISR (8-9\%), jet energy scale and resolution $(2-6 \%)$, and $p_{\mathrm{T}}^{\text {miss }}(0.4 \%)$, with the values of these uncertainties depending on chargino mass and lifetime. We also estimate uncertainties in the efficiency of the selection criteria on missing inner, middle, and outer hits (1-3, 0.3-3, and 0-3\%, respectively), and $E_{\text {calo }}(0.6-1 \%)$, with values that depend on the run period being considered. We evaluate uncertainties to account for potential mismodeling of the trigger efficiency (4-6\%, depending on chargino mass and lifetime) and track reconstruction efficiency, namely, 1.5 (4.5)\% for 2015 (2016) data. The systematic uncertainties in the signal yields are summarized in table 3 .

\section{Results}

The numbers of expected events from background sources compared with the observation in the search sample are shown in table 4 . The observation agrees with the expected 


\begin{tabular}{|lcccc|}
\hline \multirow{2}{*}{ Run period } & \multicolumn{2}{c}{ Estimated number of background events } & \multirow{2}{*}{ Observed events } \\
& Leptons & Spurious tracks & Total & \\
\hline 2015 & $0.1 \pm 0.1$ & $0_{-0}^{+0.1}$ & $0.1 \pm 0.1$ & 1 \\
2016A & $2.0 \pm 0.4 \pm 0.1$ & $0.4 \pm 0.2 \pm 0.4$ & $2.4 \pm 0.5 \pm 0.4$ & 2 \\
2016B & $3.1 \pm 0.6 \pm 0.2$ & $0.9 \pm 0.4 \pm 0.9$ & $4.0 \pm 0.7 \pm 0.9$ & 4 \\
Total & $5.2 \pm 0.8 \pm 0.3$ & $1.3 \pm 0.4 \pm 1.0$ & $6.5 \pm 0.9 \pm 1.0$ & 7 \\
\hline
\end{tabular}

Table 4. Summary of numbers of events for the estimated backgrounds and the observed data. The uncertainties include those from statistical and systematic sources. In categories where the systematic uncertainty is negligible, it is not shown.

background. We set 95\% CL upper limits on the product of the cross section for direct production of charginos $(\sigma)$ and their branching fraction to $\widetilde{\chi}_{1}^{0} \pi^{ \pm}(\mathcal{B})$ for various chargino masses and lifetimes.

These limits are calculated using the LHC-type [53] modified frequentist $\mathrm{CL}_{\mathrm{s}}$ criterion $[54,55]$. This method uses a test statistic based on a profile likelihood ratio [56] and treats nuisance parameters in a frequentist context. Nuisance parameters for the theoretical uncertainties in the signal cross sections, and systematic uncertainties in the integrated luminosity and in the signal selection efficiency, are constrained with log-normal distributions. There are two types of nuisance parameters for the uncertainties in the background estimates, and they are specified separately for each of the four background contributions (three arising from the three flavors of charged leptons and one from spurious tracks). Those that result from the limited size of the control samples are constrained with gamma distributions, while those that are associated with statistical uncertainties in multiplicative factors and the systematic uncertainties discussed in section 6 are constrained with log-normal distributions.

The expected and observed limits on the product of $\sigma$ and $\mathcal{B}$ are shown in figure 3 as a function of chargino mass, for three different chargino lifetimes. Both $\widetilde{\chi}_{1}^{0} \widetilde{\chi}_{1}^{ \pm}$and $\tilde{\chi}_{1}^{ \pm} \widetilde{\chi}_{1}^{\mp}$ production are included in $\sigma$ as a function of chargino mass as given in theory, which predicts a ratio of roughly 2:1 over the masses considered. The intersection of the theoretical prediction and the upper limit on the cross section is used to set a constraint on the mass of the chargino, for a given chargino lifetime. This procedure is repeated for a large number of chargino lifetimes, in order to produce a two-dimensional constraint on the chargino mass and mean proper lifetime, which is shown in figure 4. Charginos with a lifetime of $3(7) \mathrm{ns}$ are excluded up to a mass of $715(695) \mathrm{GeV}$. Conversely, charginos with a mass of $505 \mathrm{GeV}$ are excluded for lifetimes from 0.5 to $60 \mathrm{~ns}$. Figure 5 shows the observed limits on the product of the cross section for direct production of charginos and their branching fraction to $\widetilde{\chi}_{1}^{0} \pi^{ \pm}$.

\section{Summary}

A search has been presented for long-lived charged particles that decay within the CMS detector and produce the signature of a disappearing track. In a sample of proton-proton 

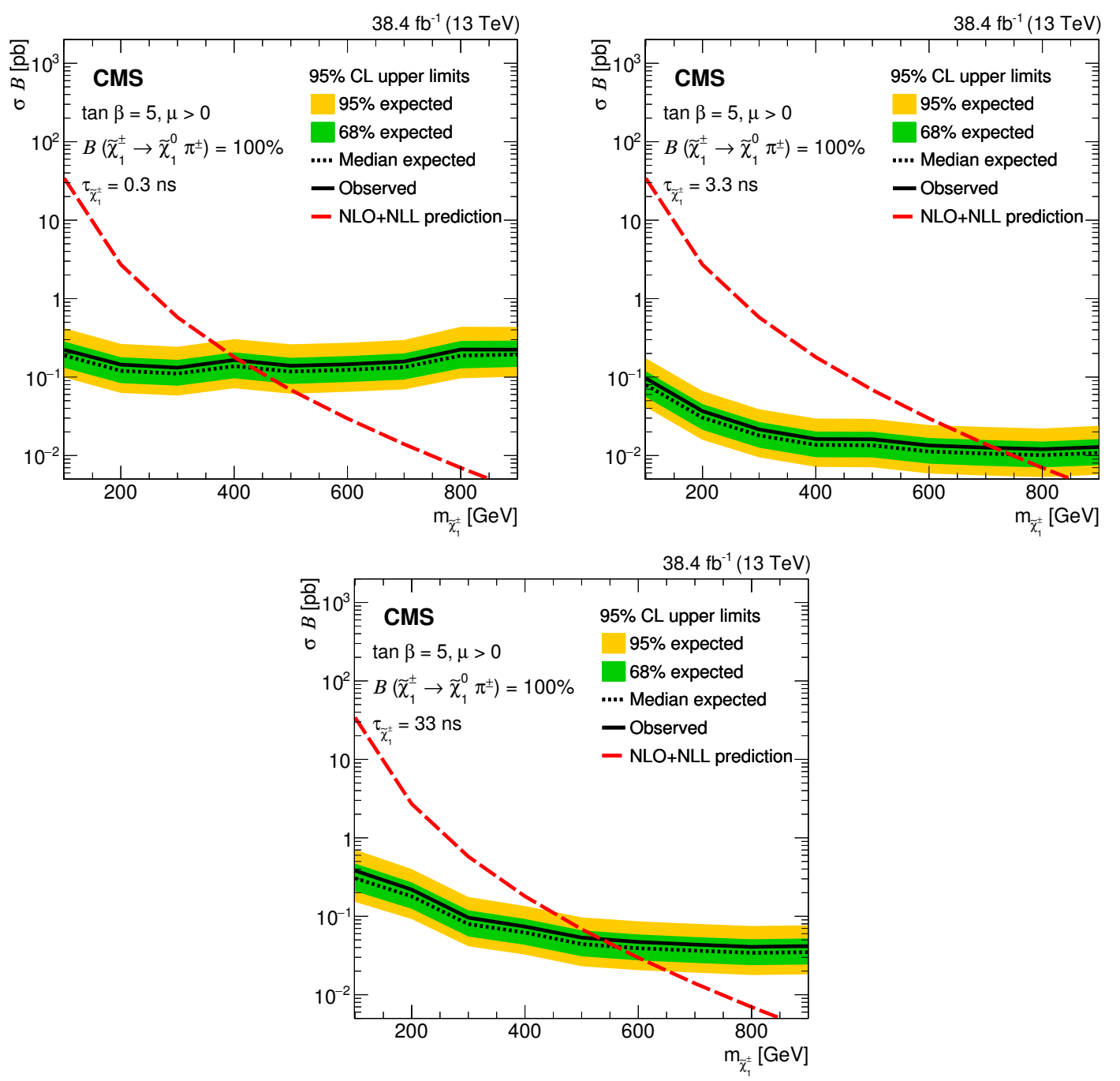

Figure 3. The expected and observed 95\% CL upper limits on the product of the cross section for direct production of charginos and their branching fraction to $\widetilde{\chi}_{1}^{0} \pi^{ \pm}$as a function of chargino mass for chargino lifetimes of $0.33,3.3$, and 33 ns. The direct chargino production cross section includes both $\widetilde{\chi}_{1}^{0} \widetilde{\chi}_{1}^{ \pm}$and $\widetilde{\chi}_{1}^{ \pm} \widetilde{\chi}_{1}^{\mp}$ production in roughly a 2:1 ratio for all chargino masses considered. The dashed red line indicates the theoretical prediction for the AMSB model.

data recorded in 2015 and 2016 at a center-of-mass energy of $13 \mathrm{TeV}$ and corresponding to an integrated luminosity of $38.4 \mathrm{fb}^{-1}$, seven events are observed, compared with the estimated background from standard model processes of $6.5 \pm 0.9$ (stat) \pm 1.0 (syst) events. The observation is consistent with the background-only hypothesis. The results are interpreted in the context of the anomaly-mediated supersymmetry breaking model, which predicts a small mass difference between the lightest chargino $\left(\widetilde{\chi}_{1}^{ \pm}\right)$and neutralino $\left(\widetilde{\chi}_{1}^{0}\right)$. The chargino decays via $\widetilde{\chi}_{1}^{ \pm} \rightarrow \widetilde{\chi}_{1}^{0} \pi^{ \pm}$, and because of the limited phase space available for the decay, the chargino has a lifetime on the order of 1 ns and the pion generally has too low momentum to yield a reconstructed track. If the chargino decays inside the tracker volume, it can thus produce a disappearing track. We place constraints on the mass of 


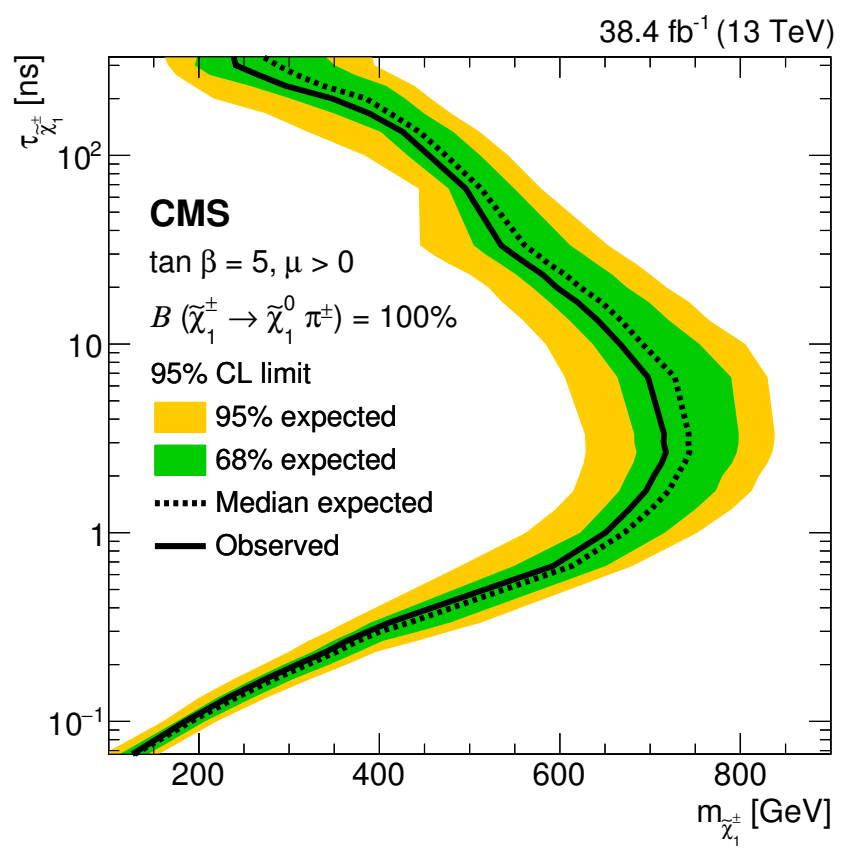

Figure 4. The expected and observed constraints on chargino lifetime and mass. The region to the left of the curve is excluded at $95 \%$ CL.

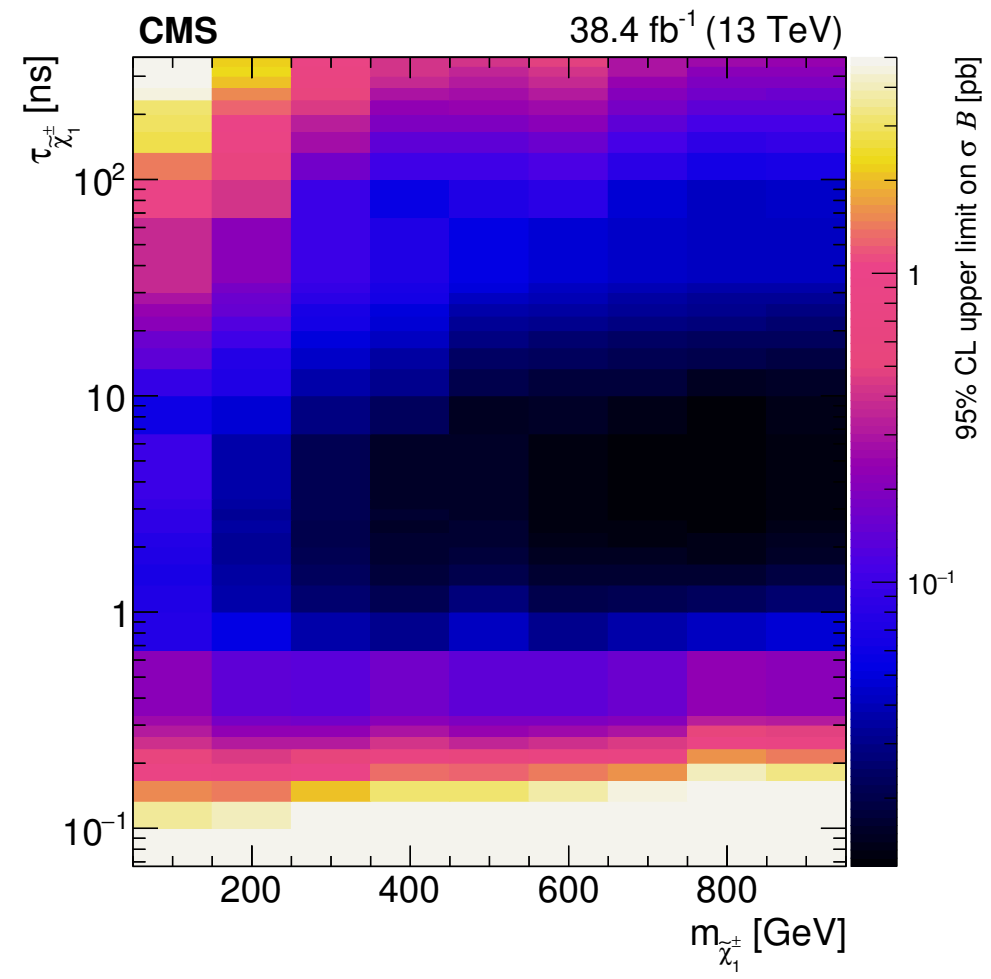

Figure 5. The observed 95\% CL upper limits on the product of the cross section for direct production of charginos and their branching fraction to $\widetilde{\chi}_{1}^{0} \pi^{ \pm}$as a function of chargino mass and lifetime. The direct chargino production cross section includes both $\widetilde{\chi}_{1}^{0} \widetilde{\chi}_{1}^{ \pm}$and $\tilde{\chi}_{1}^{ \pm} \tilde{\chi}_{1}^{\mp}$ production in roughly a 2:1 ratio for all chargino masses considered. 
charginos from direct electroweak production, for chargino mean proper lifetimes between 0.1 and $100 \mathrm{~ns}$. Charginos with masses up to 715 (695) GeV for a lifetime of 3 (7) ns are excluded at $95 \%$ confidence level, as are charginos with lifetimes from 0.5 to $60 \mathrm{~ns}$ for a mass of $505 \mathrm{GeV}$. These constraints extend the limits set by a previous search for disappearing tracks performed by the CMS collaboration [12] and are complementary to the limits set by searches for heavy stable charged particles, which exclude charginos with much longer lifetimes $[15,16]$. For chargino lifetimes above $\approx 0.7 \mathrm{~ns}$, the present search places the most stringent constraints using a disappearing track signature on direct chargino production.

\section{Acknowledgments}

We congratulate our colleagues in the CERN accelerator departments for the excellent performance of the LHC and thank the technical and administrative staffs at CERN and at other CMS institutes for their contributions to the success of the CMS effort. In addition, we gratefully acknowledge the computing centers and personnel of the Worldwide LHC Computing Grid for delivering so effectively the computing infrastructure essential to our analyses. Finally, we acknowledge the enduring support for the construction and operation of the LHC and the CMS detector provided by the following funding agencies: BMWFW and FWF (Austria); FNRS and FWO (Belgium); CNPq, CAPES, FAPERJ, and FAPESP (Brazil); MES (Bulgaria); CERN; CAS, MoST, and NSFC (China); COLCIENCIAS (Colombia); MSES and CSF (Croatia); RPF (Cyprus); SENESCYT (Ecuador); MoER, ERC IUT, and ERDF (Estonia); Academy of Finland, MEC, and HIP (Finland); CEA and CNRS/IN2P3 (France); BMBF, DFG, and HGF (Germany); GSRT (Greece); NKFIA (Hungary); DAE and DST (India); IPM (Iran); SFI (Ireland); INFN (Italy); MSIP and NRF (Republic of Korea); LAS (Lithuania); MOE and UM (Malaysia); BUAP, CINVESTAV, CONACYT, LNS, SEP, and UASLP-FAI (Mexico); MBIE (New Zealand); PAEC (Pakistan); MSHE and NSC (Poland); FCT (Portugal); JINR (Dubna); MON, RosAtom, RAS and RFBR (Russia); MESTD (Serbia); SEIDI, CPAN, PCTI and FEDER (Spain); Swiss Funding Agencies (Switzerland); MST (Taipei); ThEPCenter, IPST, STAR, and NSTDA (Thailand); TUBITAK and TAEK (Turkey); NASU and SFFR (Ukraine); STFC (United Kingdom); DOE and NSF (U.S.A.).

Individuals have received support from the Marie-Curie program and the European Research Council and Horizon 2020 Grant, contract No. 675440 (European Union); the Leventis Foundation; the A. P. Sloan Foundation; the Alexander von Humboldt Foundation; the Belgian Federal Science Policy Office; the Fonds pour la Formation à la Recherche dans l'Industrie et dans l'Agriculture (FRIA-Belgium); the Agentschap voor Innovatie door Wetenschap en Technologie (IWT-Belgium); the F.R.S.-FNRS and FWO (Belgium) under the "Excellence of Science - EOS" — be.h project n. 30820817; the Ministry of Education, Youth and Sports (MEYS) of the Czech Republic; the Lendület ("Momentum") Programme and the János Bolyai Research Scholarship of the Hungarian Academy of Sciences, the New National Excellence Program ÚNKP, the NKFIA research grants 123842, 123959, 124845, 124850 and 125105 (Hungary); the Council of Science and Industrial Research, India; the HOMING PLUS program of the Foundation for Polish Science, cofinanced from 
European Union, Regional Development Fund, the Mobility Plus program of the Ministry of Science and Higher Education, the National Science Center (Poland), contracts Harmonia 2014/14/M/ST2/00428, Opus 2014/13/B/ST2/02543, 2014/15/B/ST2/03998, and 2015/19/B/ST2/02861, Sonata-bis 2012/07/E/ST2/01406; the National Priorities Research Program by Qatar National Research Fund; the Programa Estatal de Fomento de la Investigación Científica y Técnica de Excelencia María de Maeztu, grant MDM-2015-0509 and the Programa Severo Ochoa del Principado de Asturias; the Thalis and Aristeia programs cofinanced by EU-ESF and the Greek NSRF; the Rachadapisek Sompot Fund for Postdoctoral Fellowship, Chulalongkorn University and the Chulalongkorn Academic into Its 2nd Century Project Advancement Project (Thailand); the Welch Foundation, contract C-1845; and the Weston Havens Foundation (U.S.A.).

Open Access. This article is distributed under the terms of the Creative Commons Attribution License (CC-BY 4.0), which permits any use, distribution and reproduction in any medium, provided the original author(s) and source are credited.

\section{References}

[1] G.F. Giudice, M.A. Luty, H. Murayama and R. Rattazzi, Gaugino mass without singlets, JHEP 12 (1998) 027 [hep-ph/9810442] [INSPIRE].

[2] L. Randall and R. Sundrum, Out of this world supersymmetry breaking, Nucl. Phys. B 557 (1999) 79 [hep-th/9810155] [INSPIRE].

[3] C.H. Chen, M. Drees and J.F. Gunion, A nonstandard string/SUSY scenario and its phenomenological implications, Phys. Rev. D 55 (1997) 330 [Erratum ibid. D 60 (1999) 039901] [hep-ph/9607421] [INSPIRE].

[4] H.-C. Cheng, B.A. Dobrescu and K.T. Matchev, Generic and chiral extensions of the supersymmetric standard model, Nucl. Phys. B 543 (1999) 47 [hep-ph/9811316] [inSPIRE].

[5] T. Gherghetta, G.F. Giudice and J.D. Wells, Phenomenological consequences of supersymmetry with anomaly induced masses, Nucl. Phys. B 559 (1999) 27 [hep-ph/9904378] [INSPIRE].

[6] J.L. Feng, T. Moroi, L. Randall, M. Strassler and S.-f. Su, Discovering supersymmetry at the Tevatron in wino LSP scenarios, Phys. Rev. Lett. 83 (1999) 1731 [hep-ph/9904250] [INSPIRE].

[7] M. Ibe, S. Matsumoto and T.T. Yanagida, Pure gravity mediation with $m_{3 / 2}=10-100 \mathrm{TeV}$, Phys. Rev. D 85 (2012) 095011 [arXiv:1202.2253] [INSPIRE].

[8] L.J. Hall, Y. Nomura and S. Shirai, Spread supersymmetry with Wino LSP: gluino and dark matter signals, JHEP 01 (2013) 036 [arXiv:1210.2395] [INSPIRE].

[9] A. Arvanitaki, N. Craig, S. Dimopoulos and G. Villadoro, Mini-split, JHEP 02 (2013) 126 [arXiv: 1210.0555] [INSPIRE].

[10] N. Arkani-Hamed et al., Simply unnatural supersymmetry, arXiv:1212.6971 [INSPIRE].

[11] M. Citron et al., End of the CMSSM coannihilation strip is nigh, Phys. Rev. D 87 (2013) 036012 [arXiv: 1212.2886] [INSPIRE]. 
[12] CMS collaboration, Search for disappearing tracks in proton-proton collisions at $\sqrt{s}=8$ TeV, JHEP 01 (2015) 096 [arXiv:1411.6006] [INSPIRE].

[13] ATLAS collaboration, Search for charginos nearly mass degenerate with the lightest neutralino based on a disappearing-track signature in pp collisions at $\sqrt{s}=8$ TeV with the ATLAS detector, Phys. Rev. D 88 (2013) 112006 [arXiv:1310.3675] [INSPIRE].

[14] ATLAS collaboration, Search for long-lived charginos based on a disappearing-track signature in pp collisions at $\sqrt{s}=13$ TeV with the ATLAS detector, JHEP 06 (2018) 022 [arXiv: 1712.02118] [INSPIRE].

[15] CMS collaboration, Searches for long-lived charged particles in pp collisions at $\sqrt{s}=7$ and 8 TeV, JHEP 07 (2013) 122 [arXiv:1305.0491] [INSPIRE].

[16] CMS collaboration, Constraints on the pMSSM, AMSB model and on other models from the search for long-lived charged particles in proton-proton collisions at $\sqrt{s}=8$ TeV, Eur. Phys. J. C 75 (2015) 325 [arXiv:1502.02522] [INSPIRE].

[17] CMS collaboration, Description and performance of track and primary-vertex reconstruction with the CMS tracker, 2014 JINST 9 P10009 [arXiv: 1405.6569] [INSPIRE].

[18] CMS collaboration, The CMS trigger system, 2017 JINST 12 P01020 [arXiv:1609.02366] [INSPIRE].

[19] CMS collaboration, The CMS experiment at the CERN LHC, 2008 JINST 3 S08004 [INSPIRE].

[20] CMS collaboration, CMS luminosity measurement for the 2015 data-taking period, CMS-PAS-LUM-15-001 (2015).

[21] CMS collaboration, CMS luminosity measurements for the 2016 data taking period, CMS-PAS-LUM-17-001 (2017).

[22] T. Sjöstrand, S. Mrenna and P.Z. Skands, PYTHIA 6.4 physics and manual, JHEP 05 (2006) 026 [hep-ph/0603175] [INSPIRE].

[23] J. Pumplin et al., New generation of parton distributions with uncertainties from global QCD analysis, JHEP 07 (2002) 012 [hep-ph/0201195] [INSPIRE].

[24] F.E. Paige, S.D. Protopopescu, H. Baer and X. Tata, ISAJET 7.69: a Monte Carlo event generator for $p p, \bar{p} p$ and $e^{+} e^{-}$reactions, hep-ph/0312045 [INSPIRE].

[25] M. Ibe, S. Matsumoto and R. Sato, Mass splitting between charged and neutral winos at two-loop level, Phys. Lett. B 721 (2013) 252 [arXiv:1212.5989] [INSPIRE].

[26] B. Fuks, M. Klasen, D.R. Lamprea and M. Rothering, Gaugino production in proton-proton collisions at a center-of-mass energy of 8 TeV, JHEP 10 (2012) 081 [arXiv:1207.2159] [INSPIRE].

[27] B. Fuks, M. Klasen, D.R. Lamprea and M. Rothering, Precision predictions for electroweak superpartner production at hadron colliders with Resummino, Eur. Phys. J. C 73 (2013) 2480 [arXiv: 1304.0790] [INSPIRE].

[28] P.M. Nadolsky et al., Implications of CTEQ global analysis for collider observables, Phys. Rev. D 78 (2008) 013004 [arXiv:0802.0007] [INSPIRE].

[29] A.D. Martin, W.J. Stirling, R.S. Thorne and G. Watt, Parton distributions for the LHC, Eur. Phys. J. C 63 (2009) 189 [arXiv:0901.0002] [INSPIRE]. 
[30] J. Butterworth et al., PDF 4 LHC recommendations for LHC run II, J. Phys. G 43 (2016) 023001 [arXiv: 1510.03865] [INSPIRE].

[31] CMS collaboration, Search for top-squark pair production in the single-lepton final state in pp collisions at $\sqrt{s}=8 \mathrm{TeV}$, Eur. Phys. J. C 73 (2013) 2677 [arXiv:1308.1586] [InSPIRE].

[32] J. Alwall et al., The automated computation of tree-level and next-to-leading order differential cross sections and their matching to parton shower simulations, JHEP 07 (2014) 079 [arXiv: 1405.0301] [inSPIRE].

[33] T. Sjöstrand, S. Mrenna and P.Z. Skands, A brief introduction to PYTHIA 8.1, Comput. Phys. Commun. 178 (2008) 852 [arXiv:0710.3820] [INSPIRE].

[34] P. Nason, A new method for combining NLO QCD with shower Monte Carlo algorithms, JHEP 11 (2004) 040 [hep-ph/0409146] [INSPIRE].

[35] S. Frixione, P. Nason and C. Oleari, Matching NLO QCD computations with Parton Shower simulations: the POWHEG method, JHEP 11 (2007) 070 [arXiv: 0709.2092] [INSPIRE].

[36] S. Alioli, P. Nason, C. Oleari and E. Re, A general framework for implementing NLO calculations in shower Monte Carlo programs: the POWHEG BOX, JHEP 06 (2010) 043 [arXiv: 1002.2581] [INSPIRE].

[37] T. Melia, P. Nason, R. Rontsch and G. Zanderighi, $W^{+} W^{-}, W Z$ and $Z Z$ production in the POWHEG BOX, JHEP 11 (2011) 078 [arXiv:1107.5051] [INSPIRE].

[38] P. Nason and G. Zanderighi, $W^{+} W^{-}, W Z$ and $Z Z$ production in the POWHEG-BOX-V2, Eur. Phys. J. C 74 (2014) 2702 [arXiv:1311.1365] [InSPIRE].

[39] S. Frixione, P. Nason and G. Ridolfi, A positive-weight next-to-leading-order Monte Carlo for heavy flavour hadroproduction, JHEP 09 (2007) 126 [arXiv:0707.3088] [INSPIRE].

[40] E. Re, Single-top Wt-channel production matched with parton showers using the POWHEG method, Eur. Phys. J. C 71 (2011) 1547 [arXiv:1009.2450] [INSPIRE].

[41] NNPDF collaboration, R.D. Ball et al., Parton distributions for the LHC Run II, JHEP 04 (2015) 040 [arXiv: 1410.8849] [INSPIRE].

[42] P. Skands, S. Carrazza and J. Rojo, Tuning PYTHIA 8.1: the Monash 2013 Tune, Eur. Phys. J. C 74 (2014) 3024 [arXiv: 1404.5630] [INSPIRE].

[43] CMS collaboration, Event generator tunes obtained from underlying event and multiparton scattering measurements, Eur. Phys. J. C 76 (2016) 155 [arXiv:1512.00815] [INSPIRE].

[44] GEANT4 collaboration, S. Agostinelli et al., GEANT4 - A simulation toolkit, Nucl. Instrum. Meth. A 506 (2003) 250 [INSPIRE].

[45] CMS collaboration, Particle-flow reconstruction and global event description with the CMS detector, 2017 JINST 12 P10003 [arXiv:1706. 04965] [INSPIRE].

[46] M. Cacciari, G.P. Salam and G. Soyez, FastJet user manual, Eur. Phys. J. C 72 (2012) 1896 [arXiv: 1111.6097] [INSPIRE].

[47] M. Cacciari, G.P. Salam and G. Soyez, The anti- $k_{t}$ jet clustering algorithm, JHEP 04 (2008) 063 [arXiv:0802.1189] [INSPIRE].

[48] CMS collaboration, Jet algorithms performance in 13 TeV data, CMS-PAS-JME-16-003 (2016). 
[49] CMS collaboration, Technical proposal for the Phase-II upgrade of the CMS detector, CERN-LHCC-2015-010 (2015).

[50] CMS collaboration, Measurements of inclusive $W$ and $Z$ cross sections in pp collisions at $\sqrt{s}=7 \mathrm{TeV}, J H E P 01$ (2011) 080 [arXiv:1012.2466] [INSPIRE].

[51] Particle Data Group, C. Patrignani et al., Review of particle physics, Chin. Phys. C 40 (2016) 100001 [INSPIRE].

[52] R.D. Cousins and V.L. Highland, Incorporating systematic uncertainties into an upper limit, Nucl. Instrum. Meth. A 320 (1992) 331 [INSPIRE].

[53] ATLAS and CMS collaborations and the LHC Higgs Combination Group, Procedure for the LHC Higgs boson search combination in Summer 2011, CMS-NOTE-2011-005 (2011).

[54] T. Junk, Confidence level computation for combining searches with small statistics, Nucl. Instrum. Meth. A 434 (1999) 435 [hep-ex/9902006] [INSPIRE].

[55] A.L. Read, Presentation of search results: the $C L_{s}$ technique, J. Phys. G 28 (2002) 2693 [INSPIRE].

[56] G. Cowan, K. Cranmer, E. Gross and O. Vitells, Asymptotic formulae for likelihood-based tests of new physics, Eur. Phys. J. C 71 (2011) 1554 [Erratum ibid. C 73 (2013) 2501] [arXiv: 1007.1727] [INSPIRE]. 


\section{The CMS collaboration}

\section{Yerevan Physics Institute, Yerevan, Armenia}

A.M. Sirunyan, A. Tumasyan

\section{Institut für Hochenergiephysik, Wien, Austria}

W. Adam, F. Ambrogi, E. Asilar, T. Bergauer, J. Brandstetter, E. Brondolin, M. Dragicevic, J. Erö, A. Escalante Del Valle, M. Flechl, R. Frühwirth ${ }^{1}$, V.M. Ghete, J. Hrubec, M. Jeitler ${ }^{1}$, N. Krammer, I. Krätschmer, D. Liko, T. Madlener, I. Mikulec, N. Rad, H. Rohringer, J. Schieck ${ }^{1}$, R. Schöfbeck, M. Spanring, D. Spitzbart, A. Taurok, W. Waltenberger, J. Wittmann, C.-E. Wulz ${ }^{1}$, M. Zarucki

Institute for Nuclear Problems, Minsk, Belarus

V. Chekhovsky, V. Mossolov, J. Suarez Gonzalez

Universiteit Antwerpen, Antwerpen, Belgium

E.A. De Wolf, D. Di Croce, X. Janssen, J. Lauwers, M. Pieters, M. Van De Klundert, H. Van Haevermaet, P. Van Mechelen, N. Van Remortel

\section{Vrije Universiteit Brussel, Brussel, Belgium}

S. Abu Zeid, F. Blekman, J. D'Hondt, I. De Bruyn, J. De Clercq, K. Deroover, G. Flouris, D. Lontkovskyi, S. Lowette, I. Marchesini, S. Moortgat, L. Moreels, Q. Python, K. Skovpen, S. Tavernier, W. Van Doninck, P. Van Mulders, I. Van Parijs

Université Libre de Bruxelles, Bruxelles, Belgium

D. Beghin, B. Bilin, H. Brun, B. Clerbaux, G. De Lentdecker, H. Delannoy, B. Dorney, G. Fasanella, L. Favart, R. Goldouzian, A. Grebenyuk, A.K. Kalsi, T. Lenzi, J. Luetic, N. Postiau, E. Starling, L. Thomas, C. Vander Velde, P. Vanlaer, D. Vannerom, Q. Wang

\section{Ghent University, Ghent, Belgium}

T. Cornelis, D. Dobur, A. Fagot, M. Gul, I. Khvastunov², D. Poyraz, C. Roskas, D. Trocino, M. Tytgat, W. Verbeke, B. Vermassen, M. Vit, N. Zaganidis

\section{Université Catholique de Louvain, Louvain-la-Neuve, Belgium}

H. Bakhshiansohi, O. Bondu, S. Brochet, G. Bruno, C. Caputo, P. David, C. Delaere, M. Delcourt, B. Francois, A. Giammanco, G. Krintiras, V. Lemaitre, A. Magitteri, A. Mertens, M. Musich, K. Piotrzkowski, A. Saggio, M. Vidal Marono, S. Wertz, J. Zobec

\section{Centro Brasileiro de Pesquisas Fisicas, Rio de Janeiro, Brazil}

F.L. Alves, G.A. Alves, L. Brito, G. Correia Silva, C. Hensel, A. Moraes, M.E. Pol, P. Rebello Teles

Universidade do Estado do Rio de Janeiro, Rio de Janeiro, Brazil

E. Belchior Batista Das Chagas, W. Carvalho, J. Chinellato ${ }^{3}$, E. Coelho, E.M. Da Costa, G.G. Da Silveira ${ }^{4}$, D. De Jesus Damiao, C. De Oliveira Martins, S. Fonseca De Souza, H. Malbouisson, D. Matos Figueiredo, M. Melo De Almeida, C. Mora Herrera, L. Mundim, H. Nogima, W.L. Prado Da Silva, L.J. Sanchez Rosas, A. Santoro, A. Sznajder, M. Thiel, E.J. Tonelli Manganote ${ }^{3}$, F. Torres Da Silva De Araujo, A. Vilela Pereira 
Universidade Estadual Paulista ${ }^{a}$, Universidade Federal do $\mathbf{A B C}^{b}$, São Paulo, Brazil

S. Ahuja ${ }^{a}$, C.A. Bernardes ${ }^{a}$, L. Calligaris $^{a}$, T.R. Fernandez Perez Tomei ${ }^{a}$, E.M. Gregores $^{b}{ }$ P.G. Mercadante ${ }^{b}$, S.F. Novaes ${ }^{a}$, Sandra S. Padula ${ }^{a}$, D. Romero $\operatorname{Abad}^{b}$

Institute for Nuclear Research and Nuclear Energy, Bulgarian Academy of Sciences, Sofia, Bulgaria

A. Aleksandrov, R. Hadjiiska, P. Iaydjiev, A. Marinov, M. Misheva, M. Rodozov,

M. Shopova, G. Sultanov

University of Sofia, Sofia, Bulgaria

A. Dimitrov, L. Litov, B. Pavlov, P. Petkov

Beihang University, Beijing, China

W. Fang ${ }^{5}, \mathrm{X} . \mathrm{Gao}^{5}$, L. Yuan

Institute of High Energy Physics, Beijing, China

M. Ahmad, J.G. Bian, G.M. Chen, H.S. Chen, M. Chen, Y. Chen, C.H. Jiang, D. Leggat,

H. Liao, Z. Liu, F. Romeo, S.M. Shaheen, A. Spiezia, J. Tao, C. Wang, Z. Wang, E. Yazgan,

H. Zhang, J. Zhao

State Key Laboratory of Nuclear Physics and Technology, Peking University, Beijing, China

Y. Ban, G. Chen, J. Li, L. Li, Q. Li, Y. Mao, S.J. Qian, D. Wang, Z. Xu

Tsinghua University, Beijing, China

Y. Wang

Universidad de Los Andes, Bogota, Colombia

C. Avila, A. Cabrera, C.A. Carrillo Montoya, L.F. Chaparro Sierra, C. Florez,

C.F. González Hernández, M.A. Segura Delgado

University of Split, Faculty of Electrical Engineering, Mechanical Engineering and Naval Architecture, Split, Croatia

B. Courbon, N. Godinovic, D. Lelas, I. Puljak, T. Sculac

University of Split, Faculty of Science, Split, Croatia

Z. Antunovic, M. Kovac

Institute Rudjer Boskovic, Zagreb, Croatia

V. Brigljevic, D. Ferencek, K. Kadija, B. Mesic, A. Starodumov ${ }^{6}$, T. Susa

University of Cyprus, Nicosia, Cyprus

M.W. Ather, A. Attikis, G. Mavromanolakis, J. Mousa, C. Nicolaou, F. Ptochos, P.A. Razis, H. Rykaczewski

Charles University, Prague, Czech Republic

M. Finger ${ }^{7}$, M. Finger Jr. ${ }^{7}$ 
Escuela Politecnica Nacional, Quito, Ecuador

E. Ayala

Universidad San Francisco de Quito, Quito, Ecuador

E. Carrera Jarrin

Academy of Scientific Research and Technology of the Arab Republic of Egypt, Egyptian Network of High Energy Physics, Cairo, Egypt

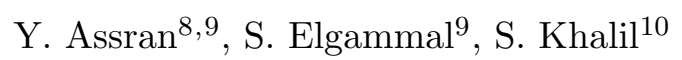

National Institute of Chemical Physics and Biophysics, Tallinn, Estonia

S. Bhowmik, A. Carvalho Antunes De Oliveira, R.K. Dewanjee, K. Ehataht, M. Kadastik, M. Raidal, C. Veelken

Department of Physics, University of Helsinki, Helsinki, Finland

P. Eerola, H. Kirschenmann, J. Pekkanen, M. Voutilainen

Helsinki Institute of Physics, Helsinki, Finland

J. Havukainen, J.K. Heikkilä, T. Järvinen, V. Karimäki, R. Kinnunen, T. Lampén, K. Lassila-Perini, S. Laurila, S. Lehti, T. Lindén, P. Luukka, T. Mäenpää, H. Siikonen, E. Tuominen, J. Tuominiemi

Lappeenranta University of Technology, Lappeenranta, Finland

T. Tuuva

IRFU, CEA, Université Paris-Saclay, Gif-sur-Yvette, France

M. Besancon, F. Couderc, M. Dejardin, D. Denegri, J.L. Faure, F. Ferri, S. Ganjour,

A. Givernaud, P. Gras, G. Hamel de Monchenault, P. Jarry, C. Leloup, E. Locci, J. Malcles,

G. Negro, J. Rander, A. Rosowsky, M.Ö. Sahin, M. Titov

Laboratoire Leprince-Ringuet, Ecole polytechnique, CNRS/IN2P3, Université Paris-Saclay, Palaiseau, France

A. Abdulsalam ${ }^{11}$, C. Amendola, I. Antropov, F. Beaudette, P. Busson, C. Charlot,

R. Granier de Cassagnac, I. Kucher, S. Lisniak, A. Lobanov, J. Martin Blanco, M. Nguyen,

C. Ochando, G. Ortona, P. Pigard, R. Salerno, J.B. Sauvan, Y. Sirois, A.G. Stahl Leiton,

A. Zabi, A. Zghiche

Université de Strasbourg, CNRS, IPHC UMR 7178, F-67000 Strasbourg, France

J.-L. Agram ${ }^{12}$, J. Andrea, D. Bloch, J.-M. Brom, E.C. Chabert, V. Cherepanov, C. Collard, E. Conte ${ }^{12}$, J.-C. Fontaine ${ }^{12}$, D. Gelé, U. Goerlach, M. Jansová, A.-C. Le Bihan, N. Tonon, P. Van Hove

Centre de Calcul de l'Institut National de Physique Nucleaire et de Physique des Particules, CNRS/IN2P3, Villeurbanne, France

S. Gadrat 
Université de Lyon, Université Claude Bernard Lyon 1, CNRS-IN2P3, Institut de Physique Nucléaire de Lyon, Villeurbanne, France

S. Beauceron, C. Bernet, G. Boudoul, N. Chanon, R. Chierici, D. Contardo, P. Depasse, H. El Mamouni, J. Fay, L. Finco, S. Gascon, M. Gouzevitch, G. Grenier, B. Ille, F. Lagarde, I.B. Laktineh, H. Lattaud, M. Lethuillier, L. Mirabito, A.L. Pequegnot, S. Perries, A. Popov ${ }^{13}$, V. Sordini, M. Vander Donckt, S. Viret, S. Zhang

\section{Georgian Technical University, Tbilisi, Georgia}

T. Toriashvili ${ }^{14}$

Tbilisi State University, Tbilisi, Georgia

Z. Tsamalaidze ${ }^{7}$

RWTH Aachen University, I. Physikalisches Institut, Aachen, Germany

C. Autermann, L. Feld, M.K. Kiesel, K. Klein, M. Lipinski, M. Preuten, M.P. Rauch, C. Schomakers, J. Schulz, M. Teroerde, B. Wittmer, V. Zhukov ${ }^{13}$

RWTH Aachen University, III. Physikalisches Institut A, Aachen, Germany

A. Albert, D. Duchardt, M. Endres, M. Erdmann, T. Esch, R. Fischer, S. Ghosh, A. Güth,

T. Hebbeker, C. Heidemann, K. Hoepfner, H. Keller, S. Knutzen, L. Mastrolorenzo,

M. Merschmeyer, A. Meyer, P. Millet, S. Mukherjee, T. Pook, M. Radziej, H. Reithler,

M. Rieger, F. Scheuch, A. Schmidt, D. Teyssier

RWTH Aachen University, III. Physikalisches Institut B, Aachen, Germany

G. Flügge, O. Hlushchenko, B. Kargoll, T. Kress, A. Künsken, T. Müller, A. Nehrkorn, A. Nowack, C. Pistone, O. Pooth, H. Sert, A. Stahl ${ }^{15}$

\section{Deutsches Elektronen-Synchrotron, Hamburg, Germany}

M. Aldaya Martin, T. Arndt, C. Asawatangtrakuldee, I. Babounikau, K. Beernaert, O. Behnke, U. Behrens, A. Bermúdez Martínez, D. Bertsche, A.A. Bin Anuar, K. Borras ${ }^{16}$, V. Botta, A. Campbell, P. Connor, C. Contreras-Campana, F. Costanza, V. Danilov, A. De Wit, M.M. Defranchis, C. Diez Pardos, D. Domínguez Damiani, G. Eckerlin, T. Eichhorn, A. Elwood, E. Eren, E. Gallo ${ }^{17}$, A. Geiser, J.M. Grados Luyando, A. Grohsjean, P. Gunnellini, M. Guthoff, M. Haranko, A. Harb, J. Hauk, H. Jung, M. Kasemann, J. Keaveney, C. Kleinwort, J. Knolle, D. Krücker, W. Lange, A. Lelek, T. Lenz, K. Lipka, W. Lohmann ${ }^{18}$, R. Mankel, I.-A. Melzer-Pellmann, A.B. Meyer, M. Meyer, M. Missiroli, G. Mittag, J. Mnich, V. Myronenko, S.K. Pflitsch, D. Pitzl, A. Raspereza, M. Savitskyi, P. Saxena, P. Schütze, C. Schwanenberger, R. Shevchenko, A. Singh, N. Stefaniuk, H. Tholen, A. Vagnerini, G.P. Van Onsem, R. Walsh, Y. Wen, K. Wichmann, C. Wissing, O. Zenaiev

\section{University of Hamburg, Hamburg, Germany}

R. Aggleton, S. Bein, A. Benecke, V. Blobel, M. Centis Vignali, T. Dreyer, E. Garutti, D. Gonzalez, J. Haller, A. Hinzmann, M. Hoffmann, A. Karavdina, G. Kasieczka, R. Klanner, R. Kogler, N. Kovalchuk, S. Kurz, V. Kutzner, J. Lange, D. Marconi, J. Multhaup, M. Niedziela, D. Nowatschin, A. Perieanu, A. Reimers, O. Rieger, C. Scharf, P. Schleper, 
S. Schumann, J. Schwandt, J. Sonneveld, H. Stadie, G. Steinbrück, F.M. Stober, M. Stöver, D. Troendle, E. Usai, A. Vanhoefer, B. Vormwald

Institut für Experimentelle Teilchenphysik, Karlsruhe, Germany

M. Akbiyik, C. Barth, M. Baselga, S. Baur, E. Butz, R. Caspart, T. Chwalek, F. Colombo, W. De Boer, A. Dierlamm, N. Faltermann, B. Freund, M. Giffels, M.A. Harrendorf, F. Hartmann ${ }^{15}$, S.M. Heindl, U. Husemann, F. Kassel ${ }^{15}$, I. Katkov ${ }^{13}$, S. Kudella, H. Mildner, S. Mitra, M.U. Mozer, Th. Müller, M. Plagge, G. Quast, K. Rabbertz, M. Schröder, I. Shvetsov, G. Sieber, H.J. Simonis, R. Ulrich, S. Wayand, M. Weber, T. Weiler,

S. Williamson, C. Wöhrmann, R. Wolf

Institute of Nuclear and Particle Physics (INPP), NCSR Demokritos, Aghia Paraskevi, Greece

G. Anagnostou, G. Daskalakis, T. Geralis, A. Kyriakis, D. Loukas, G. Paspalaki, I. TopsisGiotis

National and Kapodistrian University of Athens, Athens, Greece

G. Karathanasis, S. Kesisoglou, P. Kontaxakis, A. Panagiotou, N. Saoulidou, E. Tziaferi, K. Vellidis

National Technical University of Athens, Athens, Greece

K. Kousouris, I. Papakrivopoulos, G. Tsipolitis

University of Ioánnina, Ioánnina, Greece

I. Evangelou, C. Foudas, P. Gianneios, P. Katsoulis, P. Kokkas, S. Mallios, N. Manthos,

I. Papadopoulos, E. Paradas, J. Strologas, F.A. Triantis, D. Tsitsonis

MTA-ELTE Lendület CMS Particle and Nuclear Physics Group, Eötvös Loránd University, Budapest, Hungary

M. Csanad, N. Filipovic, P. Major, M.I. Nagy, G. Pasztor, O. Surányi, G.I. Veres

Wigner Research Centre for Physics, Budapest, Hungary

G. Bencze, C. Hajdu, D. Horvath ${ }^{19}$, Á. Hunyadi, F. Sikler, T.Á. Vámi, V. Veszpremi, G. Vesztergombi ${ }^{\dagger}$

Institute of Nuclear Research ATOMKI, Debrecen, Hungary

N. Beni, S. Czellar, J. Karancsi21 ${ }^{21}$ A. Makovec, J. Molnar, Z. Szillasi

Institute of Physics, University of Debrecen, Debrecen, Hungary

M. Bartók ${ }^{20}$, P. Raics, Z.L. Trocsanyi, B. Ujvari

Indian Institute of Science (IISc), Bangalore, India

S. Choudhury, J.R. Komaragiri, P.C. Tiwari

National Institute of Science Education and Research, Bhubaneswar, India S. Bahinipati ${ }^{22}$, P. Mal, K. Mandal, A. Nayak ${ }^{23}$, D.K. Sahoo ${ }^{22}$, S.K. Swain 
Panjab University, Chandigarh, India

S. Bansal, S.B. Beri, V. Bhatnagar, S. Chauhan, R. Chawla, N. Dhingra, R. Gupta,

A. Kaur, A. Kaur, M. Kaur, S. Kaur, R. Kumar, P. Kumari, M. Lohan, A. Mehta,

K. Sandeep, S. Sharma, J.B. Singh, G. Walia

University of Delhi, Delhi, India

A. Bhardwaj, B.C. Choudhary, R.B. Garg, M. Gola, S. Keshri, Ashok Kumar, S. Malhotra, M. Naimuddin, P. Priyanka, K. Ranjan, Aashaq Shah, R. Sharma

Saha Institute of Nuclear Physics, HBNI, Kolkata, India

R. Bhardwaj ${ }^{24}$, M. Bharti, R. Bhattacharya, S. Bhattacharya, U. Bhawandeep ${ }^{24}$, D. Bhowmik, S. Dey, S. Dutt ${ }^{24}$, S. Dutta, S. Ghosh, K. Mondal, S. Nandan, A. Purohit, P.K. Rout, A. Roy, S. Roy Chowdhury, S. Sarkar, M. Sharan, B. Singh, S. Thakur ${ }^{24}$

Indian Institute of Technology Madras, Madras, India

P.K. Behera

Bhabha Atomic Research Centre, Mumbai, India

R. Chudasama, D. Dutta, V. Jha, V. Kumar, P.K. Netrakanti, L.M. Pant, P. Shukla

Tata Institute of Fundamental Research-A, Mumbai, India

T. Aziz, M.A. Bhat, S. Dugad, G.B. Mohanty, N. Sur, B. Sutar, Ravindra Kumar Verma

Tata Institute of Fundamental Research-B, Mumbai, India

S. Banerjee, S. Bhattacharya, S. Chatterjee, P. Das, M. Guchait, Sa. Jain, S. Kumar, M. Maity ${ }^{25}$, G. Majumder, K. Mazumdar, N. Sahoo, T. Sarkar ${ }^{25}$

Indian Institute of Science Education and Research (IISER), Pune, India

S. Chauhan, S. Dube, V. Hegde, A. Kapoor, K. Kothekar, S. Pandey, A. Rane, S. Sharma

Institute for Research in Fundamental Sciences (IPM), Tehran, Iran

S. Chenarani ${ }^{26}$, E. Eskandari Tadavani, S.M. Etesami ${ }^{26}$, M. Khakzad, M. Mohammadi Najafabadi, M. Naseri, F. Rezaei Hosseinabadi, B. Safarzadeh ${ }^{27}$, M. Zeinali

University College Dublin, Dublin, Ireland

M. Felcini, M. Grunewald

INFN Sezione di Bari ${ }^{a}$, Università di Bari ${ }^{b}$, Politecnico di Bari ${ }^{c}$, Bari, Italy

M. Abbrescia ${ }^{a, b}$, C. Calabria ${ }^{a, b}$, A. Colaleo ${ }^{a}$, D. Creanza ${ }^{a, c}$, L. Cristella $^{a, b}$, N. De Filippis $^{a, c}$, M. De Palma ${ }^{a, b}$, A. Di Florio ${ }^{a, b}$, F. Errico ${ }^{a, b}$, L. Fiore ${ }^{a}$, A. Gelmi $^{a, b}$, G. Iaselli ${ }^{a, c}$, S. Lezki $^{a, b}$,

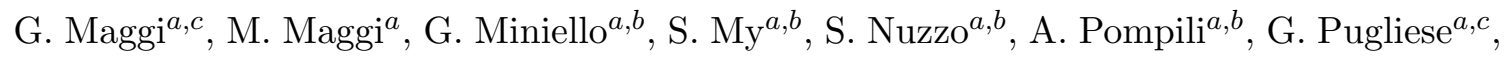
R. Radogna ${ }^{a}$, A. Ranieri ${ }^{a}$, G. Selvaggi ${ }^{a, b}$, A. Sharma ${ }^{a}$, L. Silvestris ${ }^{a, 15}$, R. Venditti ${ }^{a}$, P. Verwilligen ${ }^{a}$, G. Zito ${ }^{a}$

INFN Sezione di Bologna ${ }^{a}$, Università di Bologna ${ }^{b}$, Bologna, Italy

G. Abbiendi ${ }^{a}$, C. Battilana ${ }^{a, b}$, D. Bonacorsi ${ }^{a, b}$, L. Borgonovi $^{a, b}$, S. Braibant-Giacomelli $^{a, b}$, L. Brigliadori ${ }^{a, b}$, R. Campanini ${ }^{a, b}$, P. Capiluppi ${ }^{a, b}$, A. Castro ${ }^{a, b}$, F.R. Cavallo ${ }^{a}$, S.S. Chhibra ${ }^{a, b}$, G. Codispoti ${ }^{a, b}$, M. Cuffiani ${ }^{a, b}$, G.M. Dallavalle ${ }^{a}$, F. Fabbri ${ }^{a}$, A. Fanfani ${ }^{a, b}$, 
P. Giacomelli ${ }^{a}$, C. Grandi ${ }^{a}$, L. Guiducci ${ }^{a}, b$, S. Marcellini ${ }^{a}$, G. Masetti ${ }^{a}$, A. Montanari ${ }^{a}$,

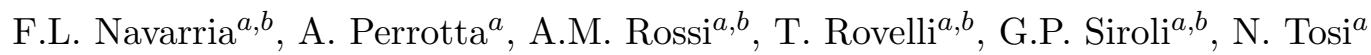

INFN Sezione di Catania ${ }^{a}$, Università di Catania ${ }^{b}$, Catania, Italy

S. Albergo ${ }^{a, b}$, A. Di Mattia ${ }^{a}$, R. Potenza ${ }^{a, b}$, A. Tricomi ${ }^{a, b}$, C. Tuve $^{a, b}$

INFN Sezione di Firenze ${ }^{a}$, Università di Firenze ${ }^{b}$, Firenze, Italy

G. Barbagli ${ }^{a}$, K. Chatterjee ${ }^{a, b}$, V. Ciulli ${ }^{a, b}$, C. Civinini ${ }^{a}$, R. D'Alessandro ${ }^{a, b}$, E. Focardi $^{a, b}{ }^{,}$

G. Latino, P. Lenzi ${ }^{a, b}$, M. Meschini ${ }^{a}$, S. Paoletti ${ }^{a}$, L. Russo $^{a}, 28$, G. Sguazzoni ${ }^{a}$, D. Strom $^{a}$,

L. Viliani ${ }^{a}$

INFN Laboratori Nazionali di Frascati, Frascati, Italy

L. Benussi, S. Bianco, F. Fabbri, D. Piccolo, F. Primavera ${ }^{15}$

INFN Sezione di Genova ${ }^{a}$, Università di Genova ${ }^{b}$, Genova, Italy

F. Ferro ${ }^{a}$, F. Ravera ${ }^{a, b}$, E. Robutti ${ }^{a}$, S. Tosi ${ }^{a, b}$

INFN Sezione di Milano-Bicocca ${ }^{a}$, Università di Milano-Bicocca ${ }^{b}$, Milano, Italy

A. Benaglia ${ }^{a}$, A. Beschi ${ }^{b}$, L. Brianza ${ }^{a, b}$, F. Brivio ${ }^{a, b}$, V. Ciriolo ${ }^{a, b, 15}$, S. Di Guida ${ }^{a, d, 15}$, M.E. Dinardo ${ }^{a, b}$, S. Fiorendi ${ }^{a, b}$, S. Gennai ${ }^{a}$, A. Ghezzi ${ }^{a, b}$, P. Govoni ${ }^{a, b}$, M. Malberti ${ }^{a, b}$, S. Malvezzi ${ }^{a}$, A. Massironi ${ }^{a, b}$, D. Menasce ${ }^{a}$, L. Moroni ${ }^{a}$, M. Paganoni ${ }^{a}, b$, D. Pedrini ${ }^{a}$, S. Ragazzi ${ }^{a, b}$, T. Tabarelli de Fatis ${ }^{a, b}$

INFN Sezione di Napoli ${ }^{a}$, Università di Napoli 'Federico II ${ }^{b}$, Napoli, Italy, Università della Basilicata $^{c}$, Potenza, Italy, Università G. Marconi ${ }^{d}$, Roma, Italy

S. Buontempo ${ }^{a}$, N. Cavallo ${ }^{a, c}$, A. Di Crescenzo ${ }^{a, b}$, F. Fabozzi $^{a, c}$, F. Fienga $^{a, b}$, G. Galati $^{a, b}$, A.O.M. Iorio ${ }^{a, b}$, W.A. $\operatorname{Khan}^{a}$, L. Lista ${ }^{a}$, S. Meola ${ }^{a, d, 15}$, P. Paolucci ${ }^{a, 15}$, C. Sciacca ${ }^{a, b}$, E. Voevodina $a^{a, b}$

INFN Sezione di Padova ${ }^{a}$, Università di Padova ${ }^{b}$, Padova, Italy, Università di Trento $^{c}$, Trento, Italy

P. Azzi ${ }^{a}$, N. Bacchetta ${ }^{a}$, L. Benato ${ }^{a, b}$, D. Bisello ${ }^{a, b}$, A. Boletti ${ }^{a, b}$, A. Bragagnolo, R. Carlin $^{a, b}$, P. Checchia ${ }^{a}$, M. Dall'Osso ${ }^{a, b}$, P. De Castro Manzano ${ }^{a}$, T. Dorigo ${ }^{a}$, F. Gasparini ${ }^{a, b}$, U. Gasparini ${ }^{a, b}$, F. Gonella ${ }^{a}$, A. Gozzelino ${ }^{a}$, S. Lacaprara ${ }^{a}$, P. Lujan, M. Margoni ${ }^{a, b}$, A.T. Meneguzzo ${ }^{a, b}$, N. Pozzobon ${ }^{a, b}$, P. Ronchese ${ }^{a, b}$, R. Rossin ${ }^{a, b}$, F. Simonetto ${ }^{a, b}$, A. Tiko, E. Torassa ${ }^{a}$, M. Zanetti ${ }^{a, b}$, P. Zotto ${ }^{a, b}$

INFN Sezione di Pavia ${ }^{a}$, Università di Pavia ${ }^{b}$, Pavia, Italy

A. Braghieri ${ }^{a}$, A. Magnani ${ }^{a}$, P. Montagna ${ }^{a, b}$, S.P. Ratti ${ }^{a, b}$, V. $\operatorname{Re}^{a}$, M. Ressegotti $^{a, b}$,

C. Riccardi ${ }^{a, b}$, P. Salvini ${ }^{a}$, I. Vai ${ }^{a, b}$, P. Vitulo ${ }^{a, b}$

INFN Sezione di Perugia ${ }^{a}$, Università di Perugia ${ }^{b}$, Perugia, Italy

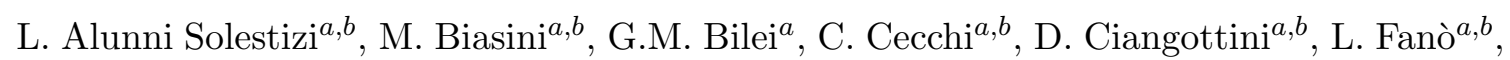
P. Lariccia ${ }^{a, b}$, E. Manoni ${ }^{a}$, G. Mantovani ${ }^{a, b}$, V. Mariani ${ }^{a, b}$, M. Menichelli ${ }^{a}$, A. Rossi ${ }^{a, b}$, A. Santocchia ${ }^{a, b}$, D. Spiga ${ }^{a}$ 
INFN Sezione di Pisa ${ }^{a}$, Università di $\mathrm{Pisa}^{b}$, Scuola Normale Superiore di Pisa ${ }^{c}$, Pisa, Italy

K. Androsov ${ }^{a}$, P. Azzurri ${ }^{a}$, G. Bagliesi ${ }^{a}$, L. Bianchini ${ }^{a}$, T. Boccali ${ }^{a}$, L. Borrello, R. Castaldi $^{a}$, M.A. Ciocci ${ }^{a, b}$, R. Dell'Orso ${ }^{a}$, G. Fedi ${ }^{a}$, L. Giannini ${ }^{a, c}$, A. Giassi ${ }^{a}$, M.T. Grippo ${ }^{a}$, F. Ligabue ${ }^{a, c}$, E. Manca ${ }^{a, c}$, G. Mandorli ${ }^{a, c}$, A. Messineo ${ }^{a, b}$, F. Palla ${ }^{a}$,

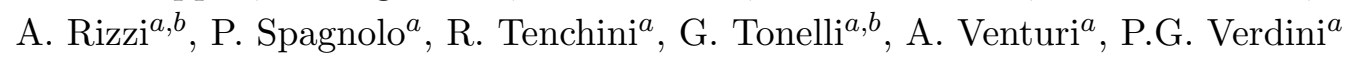

INFN Sezione di Roma ${ }^{a}$, Sapienza Università di Roma ${ }^{b}$, Rome, Italy

L. Barone ${ }^{a, b}$, F. Cavallari $^{a}$, M. Cipriani ${ }^{a, b}$, N. Daci ${ }^{a}$, D. Del Re ${ }^{a, b}$, E. Di Marco ${ }^{a, b}$, M. Diemoz ${ }^{a}$, S. Gelli ${ }^{a, b}$, E. Longo ${ }^{a, b}$, B. Marzocchi ${ }^{a, b}$, P. Meridiani ${ }^{a}$, G. Organtini ${ }^{a, b}$,

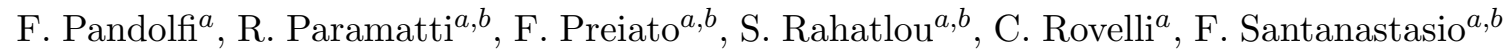

INFN Sezione di Torino ${ }^{a}$, Università di Torino ${ }^{b}$, Torino, Italy, Università del Piemonte Orientale ${ }^{c}$, Novara, Italy

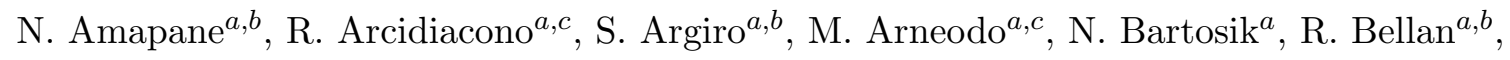

C. Biino ${ }^{a}$, N. Cartiglia ${ }^{a}$, F. Cenna ${ }^{a, b}$, S. Cometti, M. Costa ${ }^{a, b}$, R. Covarelli ${ }^{a, b}$, N. Demaria $^{a}$,

B. Kiani ${ }^{a, b}$, C. Mariotti ${ }^{a}$, S. Maselli ${ }^{a}$, E. Migliore ${ }^{a, b}$, V. Monaco ${ }^{a, b}$, E. Monteil ${ }^{a, b}$, M. Monteno ${ }^{a}$, M.M. Obertino ${ }^{a, b}$, L. Pacher ${ }^{a, b}$, N. Pastrone ${ }^{a}$, M. Pelliccioni ${ }^{a}$, G.L. Pinna

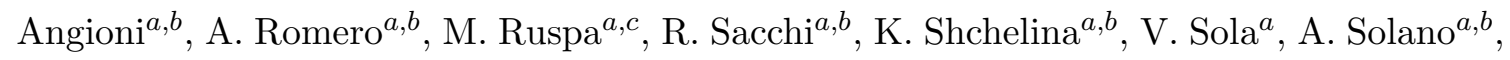
D. Soldi, A. Staiano ${ }^{a}$

INFN Sezione di Trieste ${ }^{a}$, Università di Trieste ${ }^{b}$, Trieste, Italy

S. Belforte ${ }^{a}$, V. Candelise ${ }^{a, b}$, M. Casarsa ${ }^{a}$, F. Cossutti $^{a}$, G. Della Ricca ${ }^{a, b}$, F. Vazzoler ${ }^{a, b}$, A. Zanetti ${ }^{a}$

Kyungpook National University

D.H. Kim, G.N. Kim, M.S. Kim, J. Lee, S. Lee, S.W. Lee, C.S. Moon, Y.D. Oh, S. Sekmen, D.C. Son, Y.C. Yang

Chonnam National University, Institute for Universe and Elementary Particles, Kwangju, Korea

H. Kim, D.H. Moon, G. Oh

Hanyang University, Seoul, Korea

J. Goh, T.J. Kim

Korea University, Seoul, Korea

S. Cho, S. Choi, Y. Go, D. Gyun, S. Ha, B. Hong, Y. Jo, K. Lee, K.S. Lee, S. Lee, J. Lim, S.K. Park, Y. Roh

Sejong University, Seoul, Korea

H. S. Kim

Seoul National University, Seoul, Korea

J. Almond, J. Kim, J.S. Kim, H. Lee, K. Lee, K. Nam, S.B. Oh, B.C. Radburn-Smith, S.h. Seo, U.K. Yang, H.D. Yoo, G.B. Yu

University of Seoul, Seoul, Korea

D. Jeon, H. Kim, J.H. Kim, J.S.H. Lee, I.C. Park 
Sungkyunkwan University, Suwon, Korea

Y. Choi, C. Hwang, J. Lee, I. Yu

Vilnius University, Vilnius, Lithuania

V. Dudenas, A. Juodagalvis, J. Vaitkus

National Centre for Particle Physics, Universiti Malaya, Kuala Lumpur, Malaysia

I. Ahmed, Z.A. Ibrahim, M.A.B. Md Ali ${ }^{29}$, F. Mohamad Idris ${ }^{30}$, W.A.T. Wan Abdullah, M.N. Yusli, Z. Zolkapli

Centro de Investigacion y de Estudios Avanzados del IPN, Mexico City, Mexico Duran-Osuna, M. C., H. Castilla-Valdez, E. De La Cruz-Burelo, Ramirez-Sanchez, G., I. Heredia-De La Cruz ${ }^{31}$, Rabadan-Trejo, R. I., R. Lopez-Fernandez, J. Mejia Guisao, Reyes-Almanza, R, A. Sanchez-Hernandez

Universidad Iberoamericana, Mexico City, Mexico

S. Carrillo Moreno, C. Oropeza Barrera, F. Vazquez Valencia

Benemerita Universidad Autonoma de Puebla, Puebla, Mexico

J. Eysermans, I. Pedraza, H.A. Salazar Ibarguen, C. Uribe Estrada

Universidad Autónoma de San Luis Potosí, San Luis Potosí, Mexico

A. Morelos Pineda

University of Auckland, Auckland, New Zealand

D. Krofcheck

University of Canterbury, Christchurch, New Zealand

S. Bheesette, P.H. Butler

National Centre for Physics, Quaid-I-Azam University, Islamabad, Pakistan

A. Ahmad, M. Ahmad, M.I. Asghar, Q. Hassan, H.R. Hoorani, A. Saddique, M.A. Shah, M. Shoaib, M. Waqas

National Centre for Nuclear Research, Swierk, Poland

H. Bialkowska, M. Bluj, B. Boimska, T. Frueboes, M. Górski, M. Kazana, K. Nawrocki, M. Szleper, P. Traczyk, P. Zalewski

Institute of Experimental Physics, Faculty of Physics, University of Warsaw, Warsaw, Poland

K. Bunkowski, A. Byszuk ${ }^{32}$, K. Doroba, A. Kalinowski, M. Konecki, J. Krolikowski, M. Misiura, M. Olszewski, A. Pyskir, M. Walczak

Laboratório de Instrumentação e Física Experimental de Partículas, Lisboa, Portugal

P. Bargassa, C. Beirão Da Cruz E Silva, A. Di Francesco, P. Faccioli, B. Galinhas, M. Gallinaro, J. Hollar, N. Leonardo, L. Lloret Iglesias, M.V. Nemallapudi, J. Seixas, G. Strong, O. Toldaiev, D. Vadruccio, J. Varela 
Joint Institute for Nuclear Research, Dubna, Russia

V. Alexakhin, A. Golunov, I. Golutvin, N. Gorbounov, I. Gorbunov, A. Kamenev, V. Karjavin, A. Lanev, A. Malakhov, V. Matveev ${ }^{33,34}$, P. Moisenz, V. Palichik, V. Perelygin, M. Savina, S. Shmatov, S. Shulha, N. Skatchkov, V. Smirnov, A. Zarubin

Petersburg Nuclear Physics Institute, Gatchina (St. Petersburg), Russia

V. Golovtsov, Y. Ivanov, V. Kim ${ }^{35}$, E. Kuznetsova ${ }^{36}$, P. Levchenko, V. Murzin, V. Oreshkin, I. Smirnov, D. Sosnov, V. Sulimov, L. Uvarov, S. Vavilov, A. Vorobyev

Institute for Nuclear Research, Moscow, Russia

Yu. Andreev, A. Dermenev, S. Gninenko, N. Golubev, A. Karneyeu, M. Kirsanov, N. Krasnikov, A. Pashenkov, D. Tlisov, A. Toropin

Institute for Theoretical and Experimental Physics, Moscow, Russia

V. Epshteyn, V. Gavrilov, N. Lychkovskaya, V. Popov, I. Pozdnyakov, G. Safronov, A. Spiridonov, A. Stepennov, V. Stolin, M. Toms, E. Vlasov, A. Zhokin

Moscow Institute of Physics and Technology, Moscow, Russia

T. Aushev, A. Bylinkin ${ }^{34}$

National Research Nuclear University 'Moscow Engineering Physics Institute' (MEPhI), Moscow, Russia

M. Chadeeva ${ }^{37}$, P. Parygin, D. Philippov, S. Polikarpov, E. Popova, V. Rusinov

P.N. Lebedev Physical Institute, Moscow, Russia

V. Andreev, M. Azarkin ${ }^{34}$, I. Dremin ${ }^{34}$, M. Kirakosyan ${ }^{34}$, S.V. Rusakov, A. Terkulov

Skobeltsyn Institute of Nuclear Physics, Lomonosov Moscow State University, Moscow, Russia

A. Baskakov, A. Belyaev, E. Boos, M. Dubinin ${ }^{38}$, L. Dudko, A. Ershov, A. Gribushin, V. Klyukhin, O. Kodolova, I. Lokhtin, I. Miagkov, S. Obraztsov, S. Petrushanko, V. Savrin, A. Snigirev

Novosibirsk State University (NSU), Novosibirsk, Russia

V. Blinov ${ }^{39}$, T. Dimova ${ }^{39}$, L. Kardapoltsev ${ }^{39}$, D. Shtol ${ }^{39}$, Y. Skovpen ${ }^{39}$

State Research Center of Russian Federation, Institute for High Energy Physics of NRC 'Kurchatov Institute', Protvino, Russia

I. Azhgirey, I. Bayshev, S. Bitioukov, D. Elumakhov, A. Godizov, V. Kachanov, A. Kalinin, D. Konstantinov, P. Mandrik, V. Petrov, R. Ryutin, S. Slabospitskii, A. Sobol, S. Troshin,

N. Tyurin, A. Uzunian, A. Volkov

National Research Tomsk Polytechnic University, Tomsk, Russia

A. Babaev, S. Baidali

University of Belgrade, Faculty of Physics and Vinca Institute of Nuclear Sciences, Belgrade, Serbia

P. Adzic ${ }^{40}$, P. Cirkovic, D. Devetak, M. Dordevic, J. Milosevic 
Centro de Investigaciones Energéticas Medioambientales y Tecnológicas (CIEMAT), Madrid, Spain

J. Alcaraz Maestre, A. Álvarez Fernández, I. Bachiller, M. Barrio Luna, J.A. Brochero Cifuentes, M. Cerrada, N. Colino, B. De La Cruz, A. Delgado Peris, C. Fernandez Bedoya, J.P. Fernández Ramos, J. Flix, M.C. Fouz, O. Gonzalez Lopez, S. Goy Lopez, J.M. Hernandez, M.I. Josa, D. Moran, A. Pérez-Calero Yzquierdo, J. Puerta Pelayo,

I. Redondo, L. Romero, M.S. Soares, A. Triossi

Universidad Autónoma de Madrid, Madrid, Spain

C. Albajar, J.F. de Trocóniz

\section{Universidad de Oviedo, Oviedo, Spain}

J. Cuevas, C. Erice, J. Fernandez Menendez, S. Folgueras, I. Gonzalez Caballero, J.R. González Fernández, E. Palencia Cortezon, V. Rodríguez Bouza, S. Sanchez Cruz, P. Vischia, J.M. Vizan Garcia

Instituto de Física de Cantabria (IFCA), CSIC-Universidad de Cantabria, Santander, Spain

I.J. Cabrillo, A. Calderon, B. Chazin Quero, J. Duarte Campderros, M. Fernandez, P.J. Fernández Manteca, A. García Alonso, J. Garcia-Ferrero, G. Gomez, A. Lopez Virto, J. Marco, C. Martinez Rivero, P. Martinez Ruiz del Arbol, F. Matorras, J. Piedra Gomez, C. Prieels, T. Rodrigo, A. Ruiz-Jimeno, L. Scodellaro, N. Trevisani, I. Vila, R. Vilar Cortabitarte

\section{CERN, European Organization for Nuclear Research, Geneva, Switzerland}

D. Abbaneo, B. Akgun, E. Auffray, P. Baillon, A.H. Ball, D. Barney, J. Bendavid, M. Bianco, A. Bocci, C. Botta, T. Camporesi, M. Cepeda, G. Cerminara, E. Chapon, Y. Chen, G. Cucciati, D. d'Enterria, A. Dabrowski, V. Daponte, A. David, A. De Roeck, N. Deelen, M. Dobson, T. du Pree, M. Dünser, N. Dupont, A. Elliott-Peisert, P. Everaerts, F. Fallavollita ${ }^{41}$, D. Fasanella, G. Franzoni, J. Fulcher, W. Funk, D. Gigi, A. Gilbert, K. Gill, F. Glege, D. Gulhan, J. Hegeman, V. Innocente, A. Jafari, P. Janot, O. Karacheban ${ }^{18}$, J. Kieseler, A. Kornmayer, M. Krammer ${ }^{1}$, C. Lange, P. Lecoq, C. Lourenço, L. Malgeri, M. Mannelli, F. Meijers, J.A. Merlin, S. Mersi, E. Meschi, P. Milenovic ${ }^{42}$, F. Moortgat, M. Mulders, J. Ngadiuba, S. Orfanelli, L. Orsini, F. Pantaleo ${ }^{15}$, L. Pape, E. Perez, M. Peruzzi, A. Petrilli, G. Petrucciani, A. Pfeiffer, M. Pierini, F.M. Pitters, D. Rabady, A. Racz, T. Reis, G. Rolandi ${ }^{43}$, M. Rovere, H. Sakulin, C. Schäfer, C. Schwick, M. Seidel, M. Selvaggi, A. Sharma, P. Silva, P. Sphicas ${ }^{44}$, A. Stakia, J. Steggemann, M. Tosi, D. Treille, A. Tsirou, V. Veckalns ${ }^{45}$, W.D. Zeuner

\section{Paul Scherrer Institut, Villigen, Switzerland}

W. Bertl ${ }^{\dagger}$, L. Caminada ${ }^{46}$, K. Deiters, W. Erdmann, R. Horisberger, Q. Ingram, H.C. Kaestli, D. Kotlinski, U. Langenegger, T. Rohe, S.A. Wiederkehr 
ETH Zurich - Institute for Particle Physics and Astrophysics (IPA), Zurich, Switzerland

M. Backhaus, L. Bäni, P. Berger, N. Chernyavskaya, G. Dissertori, M. Dittmar, M. Donegà, C. Dorfer, C. Grab, C. Heidegger, D. Hits, J. Hoss, T. Klijnsma, W. Lustermann, R.A. Manzoni, M. Marionneau, M.T. Meinhard, D. Meister, F. Micheli, P. Musella, F. Nessi-Tedaldi, J. Pata, F. Pauss, G. Perrin, L. Perrozzi, S. Pigazzini, M. Quittnat, M. Reichmann, D. Ruini, D.A. Sanz Becerra, M. Schönenberger, L. Shchutska, V.R. Tavolaro, K. Theofilatos, M.L. Vesterbacka Olsson, R. Wallny, D.H. Zhu

Universität Zürich, Zurich, Switzerland

T.K. Aarrestad, C. Amsler ${ }^{47}$, D. Brzhechko, M.F. Canelli, A. De Cosa, R. Del Burgo, S. Donato, C. Galloni, T. Hreus, B. Kilminster, I. Neutelings, D. Pinna, G. Rauco, P. Robmann, D. Salerno, K. Schweiger, C. Seitz, Y. Takahashi, A. Zucchetta

National Central University, Chung-Li, Taiwan

Y.H. Chang, K.y. Cheng, T.H. Doan, Sh. Jain, R. Khurana, C.M. Kuo, W. Lin, A. Pozdnyakov, S.S. Yu

National Taiwan University (NTU), Taipei, Taiwan

P. Chang, Y. Chao, K.F. Chen, P.H. Chen, W.-S. Hou, Arun Kumar, Y.y. Li, R.-S. Lu, E. Paganis, A. Psallidas, A. Steen, J.f. Tsai

Chulalongkorn University, Faculty of Science, Department of Physics, Bangkok, Thailand

B. Asavapibhop, N. Srimanobhas, N. Suwonjandee

Çukurova University, Physics Department, Science and Art Faculty, Adana, Turkey

A. Bat, F. Boran, S. Cerci ${ }^{48}$, S. Damarseckin, Z.S. Demiroglu, C. Dozen, I. Dumanoglu, S. Girgis, G. Gokbulut, Y. Guler, E. Gurpinar, I. $\mathrm{Hos}^{49}$, E.E. Kangal ${ }^{50}$, O. Kara, A. Kayis Topaksu, U. Kiminsu, M. Oglakci, G. Onengut, K. Ozdemir ${ }^{51}$, S. Ozturk ${ }^{52}$, D. Sunar Cerci $^{48}$, B. Tali ${ }^{48}$, U.G. Tok, S. Turkcapar, I.S. Zorbakir, C. Zorbilmez

Middle East Technical University, Physics Department, Ankara, Turkey

B. Isildak ${ }^{53}$, G. Karapinar ${ }^{54}$, M. Yalvac, M. Zeyrek

Bogazici University, Istanbul, Turkey

I.O. Atakisi, E. Gülmez, M. Kaya ${ }^{55}$, O. Kaya ${ }^{56}$, S. Tekten, E.A. Yetkin ${ }^{57}$

Istanbul Technical University, Istanbul, Turkey

M.N. Agaras, S. Atay, A. Cakir, K. Cankocak, Y. Komurcu, S. Sen ${ }^{58}$

Institute for Scintillation Materials of National Academy of Science of Ukraine, Kharkov, Ukraine

B. Grynyov

National Scientific Center, Kharkov Institute of Physics and Technology, Kharkov, Ukraine

L. Levchuk 


\section{University of Bristol, Bristol, United Kingdom}

F. Ball, L. Beck, J.J. Brooke, D. Burns, E. Clement, D. Cussans, O. Davignon, H. Flacher, J. Goldstein, G.P. Heath, H.F. Heath, L. Kreczko, D.M. Newbold ${ }^{59}$, S. Paramesvaran, B. Penning, T. Sakuma, D. Smith, V.J. Smith, J. Taylor, A. Titterton

Rutherford Appleton Laboratory, Didcot, United Kingdom

K.W. Bell, A. Belyaev ${ }^{60}$, C. Brew, R.M. Brown, D. Cieri, D.J.A. Cockerill, J.A. Coughlan, K. Harder, S. Harper, J. Linacre, E. Olaiya, D. Petyt, C.H. Shepherd-Themistocleous, A. Thea, I.R. Tomalin, T. Williams, W.J. Womersley

\section{Imperial College, London, United Kingdom}

G. Auzinger, R. Bainbridge, P. Bloch, J. Borg, S. Breeze, O. Buchmuller, A. Bundock, S. Casasso, D. Colling, L. Corpe, P. Dauncey, G. Davies, M. Della Negra, R. Di Maria, Y. Haddad, G. Hall, G. Iles, T. James, M. Komm, C. Laner, L. Lyons, A.-M. Magnan, S. Malik, A. Martelli, J. Nash ${ }^{61}$, A. Nikitenko ${ }^{6}$, V. Palladino, M. Pesaresi, A. Richards, A. Rose, E. Scott, C. Seez, A. Shtipliyski, T. Strebler, S. Summers, A. Tapper, K. Uchida, T. Virdee ${ }^{15}$, N. Wardle, D. Winterbottom, J. Wright, S.C. Zenz

\section{Brunel University, Uxbridge, United Kingdom}

J.E. Cole, P.R. Hobson, A. Khan, P. Kyberd, C.K. Mackay, A. Morton, I.D. Reid, L. Teodorescu, S. Zahid

\section{Baylor University, Waco, U.S.A.}

K. Call, J. Dittmann, K. Hatakeyama, H. Liu, C. Madrid, B. Mcmaster, N. Pastika, C. Smith

\section{Catholic University of America, Washington DC, U.S.A.}

R. Bartek, A. Dominguez

The University of Alabama, Tuscaloosa, U.S.A.

A. Buccilli, S.I. Cooper, C. Henderson, P. Rumerio, C. West

\section{Boston University, Boston, U.S.A.}

D. Arcaro, T. Bose, D. Gastler, D. Rankin, C. Richardson, J. Rohlf, L. Sulak, D. Zou

\section{Brown University, Providence, U.S.A.}

G. Benelli, X. Coubez, D. Cutts, M. Hadley, J. Hakala, U. Heintz, J.M. Hogan ${ }^{62}$, K.H.M. Kwok, E. Laird, G. Landsberg, J. Lee, Z. Mao, M. Narain, J. Pazzini, S. Piperov, S. Sagir ${ }^{63}$, R. Syarif, D. Yu

\section{University of California, Davis, Davis, U.S.A.}

R. Band, C. Brainerd, R. Breedon, D. Burns, M. Calderon De La Barca Sanchez, M. Chertok, J. Conway, R. Conway, P.T. Cox, R. Erbacher, C. Flores, G. Funk, W. Ko, O. Kukral, R. Lander, C. Mclean, M. Mulhearn, D. Pellett, J. Pilot, S. Shalhout, M. Shi, D. Stolp, D. Taylor, K. Tos, M. Tripathi, Z. Wang, F. Zhang

\section{University of California, Los Angeles, U.S.A.}

M. Bachtis, C. Bravo, R. Cousins, A. Dasgupta, A. Florent, J. Hauser, M. Ignatenko, N. Mccoll, S. Regnard, D. Saltzberg, C. Schnaible, V. Valuev 
University of California, Riverside, Riverside, U.S.A.

E. Bouvier, K. Burt, R. Clare, J.W. Gary, S.M.A. Ghiasi Shirazi, G. Hanson, G. Karapostoli, E. Kennedy, F. Lacroix, O.R. Long, M. Olmedo Negrete, M.I. Paneva, W. Si, L. Wang, H. Wei, S. Wimpenny, B. R. Yates

\section{University of California, San Diego, La Jolla, U.S.A.}

J.G. Branson, S. Cittolin, M. Derdzinski, R. Gerosa, D. Gilbert, B. Hashemi, A. Holzner, D. Klein, G. Kole, V. Krutelyov, J. Letts, M. Masciovecchio, D. Olivito, S. Padhi, M. Pieri, M. Sani, V. Sharma, S. Simon, M. Tadel, A. Vartak, S. Wasserbaech ${ }^{64}$, J. Wood, F. Würthwein, A. Yagil, G. Zevi Della Porta

University of California, Santa Barbara - Department of Physics, Santa Barbara, U.S.A.

N. Amin, R. Bhandari, J. Bradmiller-Feld, C. Campagnari, M. Citron, A. Dishaw, V. Dutta, M. Franco Sevilla, L. Gouskos, R. Heller, J. Incandela, A. Ovcharova, H. Qu, J. Richman, D. Stuart, I. Suarez, S. Wang, J. Yoo

California Institute of Technology, Pasadena, U.S.A.

D. Anderson, A. Bornheim, J.M. Lawhorn, H.B. Newman, T. Q. Nguyen, M. Spiropulu, J.R. Vlimant, R. Wilkinson, S. Xie, Z. Zhang, R.Y. Zhu

Carnegie Mellon University, Pittsburgh, U.S.A.

M.B. Andrews, T. Ferguson, T. Mudholkar, M. Paulini, M. Sun, I. Vorobiev, M. Weinberg

\section{University of Colorado Boulder, Boulder, U.S.A.}

J.P. Cumalat, W.T. Ford, F. Jensen, A. Johnson, M. Krohn, S. Leontsinis, E. MacDonald, T. Mulholland, K. Stenson, K.A. Ulmer, S.R. Wagner

Cornell University, Ithaca, U.S.A.

J. Alexander, J. Chaves, Y. Cheng, J. Chu, A. Datta, K. Mcdermott, N. Mirman, J.R. Patterson, D. Quach, A. Rinkevicius, A. Ryd, L. Skinnari, L. Soffi, S.M. Tan, Z. Tao, J. Thom, J. Tucker, P. Wittich, M. Zientek

\section{Fermi National Accelerator Laboratory, Batavia, U.S.A.}

S. Abdullin, M. Albrow, M. Alyari, G. Apollinari, A. Apresyan, A. Apyan, S. Banerjee, L.A.T. Bauerdick, A. Beretvas, J. Berryhill, P.C. Bhat, G. Bolla ${ }^{\dagger}$, K. Burkett, J.N. Butler, A. Canepa, G.B. Cerati, H.W.K. Cheung, F. Chlebana, M. Cremonesi, J. Duarte, V.D. Elvira, J. Freeman, Z. Gecse, E. Gottschalk, L. Gray, D. Green, S. Grünendahl, O. Gutsche, J. Hanlon, R.M. Harris, S. Hasegawa, J. Hirschauer, Z. Hu, B. Jayatilaka, S. Jindariani, M. Johnson, U. Joshi, B. Klima, M.J. Kortelainen, B. Kreis, S. Lammel, D. Lincoln, R. Lipton, M. Liu, T. Liu, J. Lykken, K. Maeshima, J.M. Marraffino, D. Mason, P. McBride, P. Merkel, S. Mrenna, S. Nahn, V. O'Dell, K. Pedro, O. Prokofyev, G. Rakness, L. Ristori, A. Savoy-Navarro ${ }^{65}$, B. Schneider, E. Sexton-Kennedy, A. Soha, W.J. Spalding, L. Spiegel, S. Stoynev, J. Strait, N. Strobbe, L. Taylor, S. Tkaczyk, N.V. Tran, L. Uplegger, E.W. Vaandering, C. Vernieri, M. Verzocchi, R. Vidal, M. Wang, H.A. Weber, A. Whitbeck 
University of Florida, Gainesville, U.S.A.

D. Acosta, P. Avery, P. Bortignon, D. Bourilkov, A. Brinkerhoff, L. Cadamuro, A. Carnes, M. Carver, D. Curry, R.D. Field, S.V. Gleyzer, B.M. Joshi, J. Konigsberg, A. Korytov, P. Ma, K. Matchev, H. Mei, G. Mitselmakher, K. Shi, D. Sperka, J. Wang, S. Wang

Florida International University, Miami, U.S.A.

Y.R. Joshi, S. Linn

Florida State University, Tallahassee, U.S.A.

A. Ackert, T. Adams, A. Askew, S. Hagopian, V. Hagopian, K.F. Johnson, T. Kolberg, G. Martinez, T. Perry, H. Prosper, A. Saha, A. Santra, V. Sharma, R. Yohay

Florida Institute of Technology, Melbourne, U.S.A.

M.M. Baarmand, V. Bhopatkar, S. Colafranceschi, M. Hohlmann, D. Noonan, M. Rahmani, T. Roy, F. Yumiceva

University of Illinois at Chicago (UIC), Chicago, U.S.A.

M.R. Adams, L. Apanasevich, D. Berry, R.R. Betts, R. Cavanaugh, X. Chen, S. Dittmer, O. Evdokimov, C.E. Gerber, D.A. Hangal, D.J. Hofman, K. Jung, J. Kamin, C. Mills, I.D. Sandoval Gonzalez, M.B. Tonjes, N. Varelas, H. Wang, Z. Wu, J. Zhang

The University of Iowa, Iowa City, U.S.A.

M. Alhusseini, B. Bilki ${ }^{66}$, W. Clarida, K. Dilsiz ${ }^{67}$, S. Durgut, R.P. Gandrajula, M. Haytmyradov, V. Khristenko, J.-P. Merlo, A. Mestvirishvili, A. Moeller, J. Nachtman, H. Ogul ${ }^{68}$, Y. Onel, F. Ozok ${ }^{69}$, A. Penzo, C. Snyder, E. Tiras, J. Wetzel

Johns Hopkins University, Baltimore, U.S.A.

B. Blumenfeld, A. Cocoros, N. Eminizer, D. Fehling, L. Feng, A.V. Gritsan, W.T. Hung, P. Maksimovic, J. Roskes, U. Sarica, M. Swartz, M. Xiao, C. You

The University of Kansas, Lawrence, U.S.A.

A. Al-bataineh, P. Baringer, A. Bean, S. Boren, J. Bowen, J. Castle, S. Khalil, A. Kropivnitskaya, D. Majumder, W. Mcbrayer, M. Murray, C. Rogan, S. Sanders, E. Schmitz, J.D. Tapia Takaki, Q. Wang

Kansas State University, Manhattan, U.S.A.

A. Ivanov, K. Kaadze, D. Kim, Y. Maravin, D.R. Mendis, T. Mitchell, A. Modak, A. Mohammadi, L.K. Saini, N. Skhirtladze

Lawrence Livermore National Laboratory, Livermore, U.S.A.

F. Rebassoo, D. Wright

University of Maryland, College Park, U.S.A.

A. Baden, O. Baron, A. Belloni, S.C. Eno, Y. Feng, C. Ferraioli, N.J. Hadley, S. Jabeen, G.Y. Jeng, R.G. Kellogg, J. Kunkle, A.C. Mignerey, F. Ricci-Tam, Y.H. Shin, A. Skuja, S.C. Tonwar, K. Wong 
Massachusetts Institute of Technology, Cambridge, U.S.A.

D. Abercrombie, B. Allen, V. Azzolini, R. Barbieri, A. Baty, G. Bauer, R. Bi, S. Brandt, W. Busza, I.A. Cali, M. D'Alfonso, Z. Demiragli, G. Gomez Ceballos, M. Goncharov, P. Harris, D. Hsu, M. Hu, Y. Iiyama, G.M. Innocenti, M. Klute, D. Kovalskyi, Y.-J. Lee, A. Levin, P.D. Luckey, B. Maier, A.C. Marini, C. Mcginn, C. Mironov, S. Narayanan, X. Niu, C. Paus, C. Roland, G. Roland, G.S.F. Stephans, K. Sumorok, K. Tatar, D. Velicanu, J. Wang, T.W. Wang, B. Wyslouch, S. Zhaozhong

University of Minnesota, Minneapolis, U.S.A.

A.C. Benvenuti, R.M. Chatterjee, A. Evans, P. Hansen, S. Kalafut, Y. Kubota, Z. Lesko, J. Mans, S. Nourbakhsh, N. Ruckstuhl, R. Rusack, J. Turkewitz, M.A. Wadud

University of Mississippi, Oxford, U.S.A.

J.G. Acosta, S. Oliveros

University of Nebraska-Lincoln, Lincoln, U.S.A.

E. Avdeeva, K. Bloom, D.R. Claes, C. Fangmeier, F. Golf, R. Gonzalez Suarez, R. Kamalieddin, I. Kravchenko, J. Monroy, J.E. Siado, G.R. Snow, B. Stieger

State University of New York at Buffalo, Buffalo, U.S.A.

A. Godshalk, C. Harrington, I. Iashvili, A. Kharchilava, D. Nguyen, A. Parker, S. Rappoccio, B. Roozbahani

Northeastern University, Boston, U.S.A.

E. Barberis, C. Freer, A. Hortiangtham, D.M. Morse, T. Orimoto, R. Teixeira De Lima,

T. Wamorkar, B. Wang, A. Wisecarver, D. Wood

Northwestern University, Evanston, U.S.A.

S. Bhattacharya, O. Charaf, K.A. Hahn, N. Mucia, N. Odell, M.H. Schmitt, K. Sung, M. Trovato, M. Velasco

University of Notre Dame, Notre Dame, U.S.A.

R. Bucci, N. Dev, M. Hildreth, K. Hurtado Anampa, C. Jessop, D.J. Karmgard, N. Kellams, K. Lannon, W. Li, N. Loukas, N. Marinelli, F. Meng, C. Mueller, Y. Musienko ${ }^{33}$, M. Planer, A. Reinsvold, R. Ruchti, P. Siddireddy, G. Smith, S. Taroni, M. Wayne, A. Wightman, M. Wolf, A. Woodard

The Ohio State University, Columbus, U.S.A.

J. Alimena, L. Antonelli, B. Bylsma, L.S. Durkin, S. Flowers, B. Francis, A. Hart, C. Hill, W. Ji, T.Y. Ling, W. Luo, B.L. Winer, H.W. Wulsin

Princeton University, Princeton, U.S.A.

S. Cooperstein, P. Elmer, J. Hardenbrook, P. Hebda, S. Higginbotham, A. Kalogeropoulos, D. Lange, M.T. Lucchini, J. Luo, D. Marlow, K. Mei, I. Ojalvo, J. Olsen, C. Palmer, P. Piroué, J. Salfeld-Nebgen, D. Stickland, C. Tully

University of Puerto Rico, Mayaguez, U.S.A.

S. Malik, S. Norberg 
Purdue University, West Lafayette, U.S.A.

A. Barker, V.E. Barnes, L. Gutay, M. Jones, A.W. Jung, A. Khatiwada, B. Mahakud, D.H. Miller, N. Neumeister, C.C. Peng, H. Qiu, J.F. Schulte, J. Sun, F. Wang, R. Xiao, W. Xie

\section{Purdue University Northwest, Hammond, U.S.A.}

T. Cheng, J. Dolen, N. Parashar

\section{Rice University, Houston, U.S.A.}

Z. Chen, K.M. Ecklund, S. Freed, F.J.M. Geurts, M. Guilbaud, M. Kilpatrick, W. Li, B. Michlin, B.P. Padley, J. Roberts, J. Rorie, W. Shi, Z. Tu, J. Zabel, A. Zhang

University of Rochester, Rochester, U.S.A.

A. Bodek, P. de Barbaro, R. Demina, Y.t. Duh, J.L. Dulemba, C. Fallon, T. Ferbel, M. Galanti, A. Garcia-Bellido, J. Han, O. Hindrichs, A. Khukhunaishvili, K.H. Lo, P. Tan, R. Taus, M. Verzetti

Rutgers, The State University of New Jersey, Piscataway, U.S.A.

A. Agapitos, J.P. Chou, Y. Gershtein, T.A. Gómez Espinosa, E. Halkiadakis, M. Heindl, E. Hughes, S. Kaplan, R. Kunnawalkam Elayavalli, S. Kyriacou, A. Lath, R. Montalvo, K. Nash, M. Osherson, H. Saka, S. Salur, S. Schnetzer, D. Sheffield, S. Somalwar, R. Stone, S. Thomas, P. Thomassen, M. Walker

University of Tennessee, Knoxville, U.S.A.

A.G. Delannoy, J. Heideman, G. Riley, K. Rose, S. Spanier, K. Thapa

Texas A\&M University, College Station, U.S.A.

O. Bouhali ${ }^{70}$, A. Castaneda Hernandez ${ }^{70}$, A. Celik, M. Dalchenko, M. De Mattia, A. Delgado, S. Dildick, R. Eusebi, J. Gilmore, T. Huang, T. Kamon ${ }^{71}$, S. Luo, R. Mueller, Y. Pakhotin, R. Patel, A. Perloff, L. Perniè, D. Rathjens, A. Safonov, A. Tatarinov

Texas Tech University, Lubbock, U.S.A.

N. Akchurin, J. Damgov, F. De Guio, P.R. Dudero, S. Kunori, K. Lamichhane, S.W. Lee, T. Mengke, S. Muthumuni, T. Peltola, S. Undleeb, I. Volobouev, Z. Wang

Vanderbilt University, Nashville, U.S.A.

S. Greene, A. Gurrola, R. Janjam, W. Johns, C. Maguire, A. Melo, H. Ni, K. Padeken, J.D. Ruiz Alvarez, P. Sheldon, S. Tuo, J. Velkovska, M. Verweij, Q. Xu

\section{University of Virginia, Charlottesville, U.S.A.}

M.W. Arenton, P. Barria, B. Cox, R. Hirosky, M. Joyce, A. Ledovskoy, H. Li, C. Neu, T. Sinthuprasith, Y. Wang, E. Wolfe, F. Xia

Wayne State University, Detroit, U.S.A.

R. Harr, P.E. Karchin, N. Poudyal, J. Sturdy, P. Thapa, S. Zaleski 


\section{University of Wisconsin - Madison, Madison, WI, U.S.A.}

M. Brodski, J. Buchanan, C. Caillol, D. Carlsmith, S. Dasu, L. Dodd, S. Duric, B. Gomber, M. Grothe, M. Herndon, A. Hervé, U. Hussain, P. Klabbers, A. Lanaro, A. Levine, K. Long, R. Loveless, T. Ruggles, A. Savin, N. Smith, W.H. Smith, N. Woods

\section{t: Deceased}

1: Also at Vienna University of Technology, Vienna, Austria

2: Also at IRFU, CEA, Université Paris-Saclay, Gif-sur-Yvette, France

3: Also at Universidade Estadual de Campinas, Campinas, Brazil

4: Also at Federal University of Rio Grande do Sul, Porto Alegre, Brazil

5: Also at Université Libre de Bruxelles, Bruxelles, Belgium

6: Also at Institute for Theoretical and Experimental Physics, Moscow, Russia

7: Also at Joint Institute for Nuclear Research, Dubna, Russia

8: Also at Suez University, Suez, Egypt

9: Now at British University in Egypt, Cairo, Egypt

10: Also at Zewail City of Science and Technology, Zewail, Egypt

11: Also at Department of Physics, King Abdulaziz University, Jeddah, Saudi Arabia

12: Also at Université de Haute Alsace, Mulhouse, France

13: Also at Skobeltsyn Institute of Nuclear Physics, Lomonosov Moscow State University, Moscow, Russia

14: Also at Tbilisi State University, Tbilisi, Georgia

15: Also at CERN, European Organization for Nuclear Research, Geneva, Switzerland

16: Also at RWTH Aachen University, III. Physikalisches Institut A, Aachen, Germany

17: Also at University of Hamburg, Hamburg, Germany

18: Also at Brandenburg University of Technology, Cottbus, Germany

19: Also at Institute of Nuclear Research ATOMKI, Debrecen, Hungary

20: Also at MTA-ELTE Lendület CMS Particle and Nuclear Physics Group, Eötvös Loránd University, Budapest, Hungary

21: Also at Institute of Physics, University of Debrecen, Debrecen, Hungary

22: Also at Indian Institute of Technology Bhubaneswar, Bhubaneswar, India

23: Also at Institute of Physics, Bhubaneswar, India

24: Also at Shoolini University, Solan, India

25: Also at University of Visva-Bharati, Santiniketan, India

26: Also at Isfahan University of Technology, Isfahan, Iran

27: Also at Plasma Physics Research Center, Science and Research Branch, Islamic Azad University, Tehran, Iran

28: Also at Università degli Studi di Siena, Siena, Italy

29: Also at International Islamic University of Malaysia, Kuala Lumpur, Malaysia

30: Also at Malaysian Nuclear Agency, MOSTI, Kajang, Malaysia

31: Also at Consejo Nacional de Ciencia y Tecnología, Mexico city, Mexico

32: Also at Warsaw University of Technology, Institute of Electronic Systems, Warsaw, Poland

33: Also at Institute for Nuclear Research, Moscow, Russia

34: Now at National Research Nuclear University 'Moscow Engineering Physics Institute' (MEPhI), Moscow, Russia

35: Also at St. Petersburg State Polytechnical University, St. Petersburg, Russia

36: Also at University of Florida, Gainesville, U.S.A.

37: Also at P.N. Lebedev Physical Institute, Moscow, Russia 
38: Also at California Institute of Technology, Pasadena, U.S.A.

39: Also at Budker Institute of Nuclear Physics, Novosibirsk, Russia

40: Also at Faculty of Physics, University of Belgrade, Belgrade, Serbia

41: Also at INFN Sezione di Pavia ${ }^{a}$, Università di Pavia ${ }^{b}$, Pavia, Italy

42: Also at University of Belgrade, Faculty of Physics and Vinca Institute of Nuclear Sciences, Belgrade, Serbia

43: Also at Scuola Normale e Sezione dell'INFN, Pisa, Italy

44: Also at National and Kapodistrian University of Athens, Athens, Greece

45: Also at Riga Technical University, Riga, Latvia

46: Also at Universität Zürich, Zurich, Switzerland

47: Also at Stefan Meyer Institute for Subatomic Physics (SMI), Vienna, Austria

48: Also at Adiyaman University, Adiyaman, Turkey

49: Also at Istanbul Aydin University, Istanbul, Turkey

50: Also at Mersin University, Mersin, Turkey

51: Also at Piri Reis University, Istanbul, Turkey

52: Also at Gaziosmanpasa University, Tokat, Turkey

53: Also at Ozyegin University, Istanbul, Turkey

54: Also at Izmir Institute of Technology, Izmir, Turkey

55: Also at Marmara University, Istanbul, Turkey

56: Also at Kafkas University, Kars, Turkey

57: Also at Istanbul Bilgi University, Istanbul, Turkey

58: Also at Hacettepe University, Ankara, Turkey

59: Also at Rutherford Appleton Laboratory, Didcot, United Kingdom

60: Also at School of Physics and Astronomy, University of Southampton, Southampton, United Kingdom

61: Also at Monash University, Faculty of Science, Clayton, Australia

62: Also at Bethel University, St. Paul, U.S.A.

63: Also at Karamanoğlu Mehmetbey University, Karaman, Turkey

64: Also at Utah Valley University, Orem, U.S.A.

65: Also at Purdue University, West Lafayette, U.S.A.

66: Also at Beykent University, Istanbul, Turkey

67: Also at Bingol University, Bingol, Turkey

68: Also at Sinop University, Sinop, Turkey

69: Also at Mimar Sinan University, Istanbul, Istanbul, Turkey

70: Also at Texas A\&M University at Qatar, Doha, Qatar

71: Also at Kyungpook National University, Daegu, Korea 